\title{
Resonance Effects in the Raman Optical Activity Spectrum of $\left[\operatorname{Rh}(\mathrm{en})_{3}\right]^{3+}$
}

\author{
Thomas Weymuth ${ }^{a, 1}$ \\ ${ }^{a}$ Laboratory for Physical Chemistry, ETH Zurich, \\ 8093 Zurich, Switzerland
}

September 30, 2019

\begin{abstract}
Raman optical activity spectra of $\Lambda$-tris-(ethylenediamine)-rhodium(III) ([Rh(en $\left.)_{3}\right]^{3+}$ ) have been calculated at 16 on-, near-, and off-resonant wavelengths between $290 \mathrm{~nm}$ and $800 \mathrm{~nm}$. The resulting spectra are analyzed in detail with a focus on the observed resonance effects. Since several electronically excited states are involved, the spectra are never monosignate, as is often observed in resonance Raman optical activity spectra. Most normal modes are enhanced through these resonance effects, but in several cases, de-enhancement effects are found. The molecular origins of the Raman optical activity intensity for selected normal modes are established by means of group coupling matrices. In general, this methodology allows one to produce an intuitive explanation for the intensity behavior of a given normal mode. However, due to the complex electronic structure of $\left[\mathrm{Rh}(\mathrm{en})_{3}\right]^{3+}$, there are some intriguing resonance effects the origins of which could not be fully clarified in terms of group coupling effects. Therefore, simple and general rules that predict how the intensity of a specific normal mode is affected by resonance effects are difficult to devise.
\end{abstract}

\footnotetext{
${ }^{1}$ Corresponding author; e-mail: thomas.weymuth@phys.chem.ethz.ch; ORCID: 0000-0001-71027022
} 


\section{Introduction}

Chiral molecules show a small difference in the intensities of Raman scattered left and right circularly polarized light, an effect generally denoted as Raman Optical Activity (ROA). ${ }^{1}$ This effect was predicted by Barron and Buckingham in $1971^{2}$ and first observed experimentally in 1973 by Barron et al. ${ }^{3}$ (this observation was independently confirmed by Hug et al. two years later ${ }^{4}$ ). Since the intensity difference measured in ROA spectroscopy is very small, it took more than twenty years and major improvements in instrumentation techniques ${ }^{5-7}$ before it was firmly established as the routine analytical method it is today. ${ }^{8-11}$ In particular, ROA spectroscopy is often adopted to study biomolecules in their natural, aqueous environment. ${ }^{8}$ However, ROA studies on chiral metal complexes are quite scarce. ${ }^{12,13}$ The first experimental ROA spectrum of a metal complex was published in 2011 by Johanessen et $a l .,{ }^{14}$ while the first theoretical ROA spectra for chiral transition metal complexes had already been presented in 2008 by Luber and Reiher. ${ }^{15}$ The same group then presented theoretical studies on chiral Cobalt complexes, ${ }^{16,17}$ and the $\beta$ domain of rat metallothionein, which features one cadmium and two zinc atoms in its center. ${ }^{18}$ More recently, Humbert-Droz et al. published a combined theoretical and experimental study on the ROA spectrum of $\Lambda$-tris-(ethylenediamine)-rhodium(III) $\left(\left[\mathrm{Rh}(\mathrm{en})_{3}\right]^{3+}\right) \cdot{ }^{19}$ In summary, all these studies have demonstrated the great potential of ROA spectroscopy for the elucidation of the structure of chiral metal complexes.

It has been found that the ROA signal can be greatly enhanced if the wavelength of the incident laser light is in resonance with an electronic excitation of the molecule under investigation, ${ }^{1}$ an effect called resonance ROA (RROA). A corresponding theory, valid in the case of resonance with a single excited electronic state, was presented in 1996 by Nafie. ${ }^{20}$ This theory predicts the resulting spectra to be monosignate and the relative band intensities identical to the one of the corresponding Raman spectrum. These predictions were confirmed by an experimental study conducted by Vargek et al. in 1998. ${ }^{21}$ If several excited electronic states are involved, however, the situation will be much more complicated; the spectra do not have to be monosignate $^{22,23}$ and de-enhancement effects may occur. ${ }^{24}$ In 2007, Jensen et al. presented an ansatz to calculate both off- and on-resonance ROA spectra, which also allowed to include multiple excited electronic states. ${ }^{25}$ Later, also Luber et al. ${ }^{24}$ as well as Barone and coworkers ${ }^{26}$ presented other approaches capable of taking multiple excited electronic states into account.

Unfortunately, there are almost no studies investigating RROA effects in transition metal compounds (the first experimental RROA spectra for a chiral transition metal compound was presented by Merten et al. in 2010 ${ }^{27}$ ). Furthermore, detailed theoretical studies of RROA spectra involving more than one excited electronic state are 
largely missing. This paper contributes to filling this gap by presenting an in-depth study of the full-resonance and near-resonance spectra of $\left[\operatorname{Rh}(\mathrm{en})_{3}\right]^{3+}$. Thereby it also complements the detailed study published by Humbert-Droz et al. on the same compound, which focused exclusively on the off-resonance case. ${ }^{19}$

This paper is organized as follows: First, we give a short introduction into the theoretical background of the calculation of (resonance) ROA spectra. Then, in Section 3, we explain the computational methodology followed in this study. Afterwards, the results are presented in Section 4. Finally, a conclusion is given in Section 5.

\section{Theoretical Background}

The (resonance) ROA intensity $\Delta I_{p}\left(180^{\circ}\right)$ of a given normal mode $p$ in a backscattering geometry (in which case the ROA intensity is maximized ${ }^{28}$ ) can be calculated as

$$
\Delta I_{p}\left(180^{\circ}\right)=I_{p}^{R}\left(180^{\circ}\right)-I_{p}^{L}\left(180^{\circ}\right) \propto \frac{1}{c}\left(24 \beta\left(G^{\prime}\right)_{p}^{2}+8 \beta(A)_{p}^{2}\right),
$$

where $c$ is the speed of light in vacuum, $\beta\left(G^{\prime}\right)_{p}^{2}$ is the anisotropic invariant of the electric-dipole-magnetic-dipole polarizability transition tensor, and $\beta(A)_{p}^{2}$ is the anisotropic invariant of the electric-dipole-electric-quadrupole polarizability transition tensor (note that all these quantities are independent of the experimental setup). The latter two quantities are given by

$$
\beta\left(G^{\prime}\right)_{p}^{2}=\operatorname{Im}\left(\frac{\mathrm{i}}{2}\left(3 \alpha_{\alpha \beta}^{(p)} G_{\alpha \beta}^{(p) *}-\alpha_{\alpha \alpha}^{(p)} G_{\beta \beta}^{(p) *}\right)\right),
$$

and

$$
\beta(A)_{p}^{2}=\operatorname{Re}\left(\frac{1}{2} \omega \alpha_{\alpha \beta}^{(p)} \epsilon_{\alpha \gamma \delta} A_{\gamma \delta \beta}^{(p) *}\right),
$$

respectively. $\alpha_{\alpha \beta}^{(p)}$ is the $\alpha \beta$-component $(\alpha, \beta=\{x, y, z\})$ of the electric-dipoleelectric-dipole polarizability for the $p$-th normal mode, while $G_{\alpha \beta}^{(p)}$ is the $\alpha \beta$-component of the electric-dipole-magnetic-dipole polarizability for the $p$-th normal mode. The asterisk denotes the complex conjugate. $\omega$ is the frequency of the incident light, and $\epsilon_{\alpha \gamma \delta}$ is the third-rank antisymmetric unit tensor. $A_{\gamma \delta \beta}^{(p)}$ is the $\gamma \delta \beta$-component $(\gamma, \delta=\{x, y, z\})$ of the electric-dipole-electric-quadrupole polarizability for the $p$-th normal mode. Re and Im denote the real and imaginary part, respectively. Note that the Einstein summation convention has been invoked for repeated greek indices.

These polarizability tensors are then calculated as

$$
\alpha_{\alpha \beta}^{(p)} G_{\alpha \beta}^{(p)}=\left(\frac{\partial \alpha_{\alpha \beta}}{\partial Q_{p}}\right)_{0}\left(\frac{\partial G_{\alpha \beta}^{\prime}}{\partial Q_{p}}\right)_{0}
$$


and

$$
\alpha_{\alpha \beta}^{(p)} \epsilon_{\alpha \gamma \delta} A_{\gamma \delta \beta}^{(p)}=\left(\frac{\partial \alpha_{\alpha \beta}}{\partial Q_{p}}\right)_{0} \epsilon_{\alpha \gamma \delta}\left(\frac{\partial A_{\gamma \delta \beta}}{\partial Q_{p}}\right)_{0}
$$

where $Q_{p}$ is the normal mode of the $p$-th vibration and the subscript " 0 " indicates that the partial derivative is to be evaluated at the molecular equilibrium structure.

In the short-time approximation adopted in the ansatz by Jensen et al. ${ }^{25} \alpha_{\alpha \beta}, G_{\alpha \beta}^{\prime}$, and $A_{\gamma \delta \beta}$ are complex quantities:

$$
\begin{aligned}
& \alpha_{\alpha \beta}=\alpha_{\alpha \beta}^{(R)}+\mathrm{i} \alpha_{\alpha \beta}^{(I)}, \\
& G_{\alpha \beta}^{\prime}=G_{\alpha \beta}^{(R)}+\mathrm{i} G_{\alpha \beta}^{(I)},
\end{aligned}
$$

and

$$
A_{\gamma \delta \beta}=A_{\gamma \delta \beta}^{(R)}+\mathrm{i} A_{\gamma \delta \beta}^{(I)}
$$

The real and imaginary parts are formally calculated from a sum over the electronic states,

$$
\begin{aligned}
& \alpha_{\alpha \beta}^{(R)}=\sum_{n \neq 0} f^{+}\left(\omega_{n 0}, \omega, \Gamma\right) \operatorname{Re}\left(\left\langle\psi_{0}\left|\mu_{\alpha}\right| \psi_{n}\right\rangle\left\langle\psi_{n}\left|\mu_{\beta}\right| \psi_{0}\right\rangle\right), \\
& \alpha_{\alpha \beta}^{(I)}=\sum_{n \neq 0} g^{-}\left(\omega_{n 0}, \omega, \Gamma\right) \operatorname{Re}\left(\left\langle\psi_{0}\left|\mu_{\alpha}\right| \psi_{n}\right\rangle\left\langle\psi_{n}\left|\mu_{\beta}\right| \psi_{0}\right\rangle\right), \\
& G_{\alpha \beta}^{(R)}=\sum_{n \neq 0} f^{-}\left(\omega_{n 0}, \omega, \Gamma\right) \operatorname{Im}\left(\left\langle\psi_{0}\left|\mu_{\alpha}\right| \psi_{n}\right\rangle\left\langle\psi_{n}\left|m_{\beta}\right| \psi_{0}\right\rangle\right), \\
& G_{\alpha \beta}^{\prime(I)}=\sum_{n \neq 0} g^{+}\left(\omega_{n 0}, \omega, \Gamma\right) \operatorname{Im}\left(\left\langle\psi_{0}\left|\mu_{\alpha}\right| \psi_{n}\right\rangle\left\langle\psi_{n}\left|m_{\beta}\right| \psi_{0}\right\rangle\right), \\
& A_{\alpha \beta \gamma}^{(R)}=\sum_{n \neq 0} f^{+}\left(\omega_{n 0}, \omega, \Gamma\right) \operatorname{Re}\left(\left\langle\psi_{0}\left|\mu_{\alpha}\right| \psi_{n}\right\rangle\left\langle\psi_{n}\left|\Theta_{\beta \gamma}\right| \psi_{0}\right\rangle\right),
\end{aligned}
$$

and

$$
A_{\alpha \beta \gamma}^{(I)}=\sum_{n \neq 0} g^{-}\left(\omega_{n 0}, \omega, \Gamma\right) \operatorname{Re}\left(\left\langle\psi_{0}\left|\mu_{\alpha}\right| \psi_{n}\right\rangle\left\langle\psi_{n}\left|\Theta_{\beta \gamma}\right| \psi_{0}\right\rangle\right)
$$


In these equations, $\psi_{0}$ is the electronic wave function of the ground state, while $\psi_{n}$ denote the electronic wave functions of excited states. Furthermore, we require the electric dipole operator

$$
\mu_{\alpha}=-\sum_{a} r_{a \alpha}
$$

and the magnetic dipole operator

$$
m_{\alpha}=-\frac{1}{2} \sum_{a} l_{a \alpha}
$$

with $l_{a \alpha}$ being the $\alpha$-component of the angular momentum operator for electron $a$. The electric quadrupole operator

$$
\Theta_{\alpha \beta}=-\frac{1}{2} \sum_{a}\left(3 r_{a \alpha} r_{a \beta}-\delta_{\alpha \beta} r_{a}^{2}\right)
$$

The sums run over all electrons $a$. Finally, in equations (9)-(14) we have the two line shape functions

$$
f^{ \pm}\left(\omega_{n 0}, \omega, \Gamma\right)=\frac{\omega_{n 0}-\omega}{\left(\omega_{n 0}-\omega\right)^{2}+\Gamma^{2}} \pm \frac{\omega_{n 0}+\omega}{\left(\omega_{n 0}+\omega\right)^{2}+\Gamma^{2}}
$$

and

$$
g^{ \pm}\left(\omega_{n 0}, \omega, \Gamma\right)=\frac{\Gamma}{\left(\omega_{n 0}-\omega\right)^{2}+\Gamma^{2}} \pm \frac{\Gamma}{\left(\omega_{n 0}+\omega\right)^{2}+\Gamma^{2}}
$$

Here, $\omega_{n 0}$ is the excitation frequency between the ground state and the $n$-th excited state, $\omega$ is the frequency of the incident light, and $1 / \Gamma$ can be interpreted as a lifetime of the excitations. Note that by setting $\Gamma=0$ in the above equations, the standard non-resonant formulation of ROA is recovered.

\section{Computational Methodology}

In their study, ${ }^{19}$ Humbert-Droz et al. analyzed in detail the different configurations and conformations possible for $\left[\mathrm{Rh}(\mathrm{en})_{3}\right]^{3+}$. Therefore, we will not attempt to repeat such an analysis in this work. Comparison with experimental spectra showed that the so-called $\Lambda(\delta \delta \delta)$ conformation (see Fig. 1; cf. Ref. 29 for more information about this nomenclature) to be the dominant conformation. ${ }^{19}$ Therefore, we will use this sole conformation throughout our study and refer to it without the prefix " $\Lambda(\delta \delta \delta)$ ".

The structure of $\left[\mathrm{Rh}(\mathrm{en})_{3}\right]^{3+}$ was first fully optimized in $\mathrm{C}_{1}$ symmetry (see the Supporting Information for the Cartesian coordinates of the optimized structure). 
This structure optimization and all subsequent calculations were done with a development version of NWCHEM (revision 29384), ${ }^{30}$ using density functional theory in its Kohn-Sham formulation. ${ }^{31}$ (It has been verified that the ROA spectra obtained with this specific version of NWCHEM are identical to the ones obtained from the officially released NWCHEM 6.8 .1 by recalculating the spectra at $800 \mathrm{~nm}$ and $307.66 \mathrm{~nm}$ incident wavelength, respectively.) In all calculations, the PBE0 exchange-correlation functional ${ }^{32}$ and the def2-TZVP basis $\operatorname{set}^{33}$ (for all atoms) were chosen. For Rhodium, the ECP-28 effective core potential ${ }^{34}$ was employed in order to take scalar relativistic effects into account. Furthermore, advantage was taken of the resolution-of-the-identity technique for the Coulomb integrals using the corresponding auxiliary basis sets. ${ }^{35}$ Finally, the D3 semiempirical dispersion corrections $^{36}$ were adopted (with the zero-damping function).

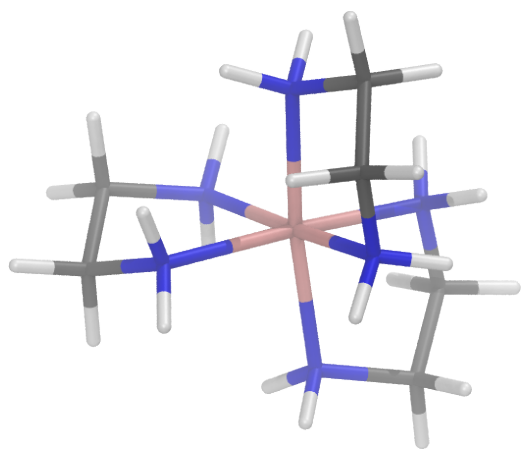

Figure 1: Structure of $\left[\mathrm{Rh}(\mathrm{en})_{3}\right]^{3+}$ investigated in this work.

All ROA spectra were calculated in the double harmonic approximation with the program suite MOVIPAC, ${ }^{37}$ relying on a seminumerical differentiation scheme with a three-step central difference formula (adopting a step size of $0.01 \mathrm{bohr}$ ). The necessary energy gradients and polarizability tensors were calculated analytically with the version of NWCHEM mentioned above relying on the ansatz by Jensen et al. ${ }^{25}$ For all spectra, a value of 0.008 a.u. was adopted for the damping parameter $\Gamma$ (we refer to the Supporting Information for a comparison of different damping values). Furthermore, great care was taken to ensure origin independence of the spectra by placing all structures close to the origin and using gauge-including atomic orbitals; note that for resonance ROA calculations, gauge-including atomic orbitals alone cannot always guarantee complete origin independence ${ }^{23}$ (see the Supporting Information for calculations verifying the gauge independence in our case). Electronic (singlet) excitations were calculated with time-dependent density functional theory employing the methodology detailed above. This yielded the lowest excita- 
tion at $307.66 \mathrm{~nm}$. Therefore, this wavelength was used in order to calculate the full-resonance spectrum. In order to investigate how this spectrum changes with different wavelengths, spectra were also calculated for the following wavelengths: $290 \mathrm{~nm}, 303 \mathrm{~nm}, 305 \mathrm{~nm}, 310 \mathrm{~nm}, 315 \mathrm{~nm}, 320 \mathrm{~nm}, 325 \mathrm{~nm}, 330 \mathrm{~nm}, 350 \mathrm{~nm}, 370 \mathrm{~nm}$, $410 \mathrm{~nm}, 500 \mathrm{~nm}, 600 \mathrm{~nm}, 700 \mathrm{~nm}$, and $800 \mathrm{~nm}$. Solvent effects were not considered in this study, as this allows us to analyze the genuine ROA spectra and of $\left[\mathrm{Rh}(\mathrm{en})_{3}\right]^{3+}$ itself and the resonance effects stemming from this isolated structure. Furthermore, as we will see below, solvent effects do not appear to play a major role in this ROA spectrum. A complete input file used to calculate the ROA spectra is given in the Supporting Information.

All line spectra were convoluted with a Lorentzian band having a full width at half maximum of $15 \mathrm{~cm}^{-1}$. This line broadening as well as the subsequent analysis of all ROA spectra was carried out with MAThematica 11.2.0. ${ }^{38}$ Plots of ROA spectra were created with MatplotliB 2.2.3. ${ }^{39}$ In these plots, the intensities of the line spectra were always scaled by a factor of 0.04. Pictures of molecular structures and molecular orbitals were generated with VMD 1.9.2. ${ }^{40,41}$ Representations of normal modes were produced with JMOL 14.6.4. ${ }^{42}$

\section{Results}

\subsection{Electronic Excitations}

To assess at which wavelength a full-resonance ROA spectrum can be calculated, we need to know the energies of the electronic excitations. The calculated energies and wavelengths of the lowest five electronic (singlet) excitations are collected in Table 1. 
Table 1: Excitation energies $E$ and corresponding wavelengths $\lambda$ of the lowest five electronic excitations as well as the molecular orbital transitions involved with a contribution larger than 0.4 a.u. to the excitation vector (transitions with a contribution larger than 0.6 a.u. are printed in boldface).

\begin{tabular}{|c|c|c|c|}
\hline No. & $E / \mathrm{eV}$ & $\lambda / \mathrm{nm}$ & "Dominating Transitions \\
\hline 1 & 4.0299 & 307.6607 & $\begin{array}{l}\text { HOMO } \rightarrow \text { LUMO } \\
\mathrm{HOMO}-1 \rightarrow \mathrm{LUMO}+1, \\
\mathrm{HOMO}-2 \rightarrow \mathrm{LUMO}\end{array}$ \\
\hline 2 & 4.0301 & 307.6455 & $\begin{array}{l}\text { HOMO } \rightarrow \text { LUMO }+\mathbf{1} \\
\mathrm{HOMO}-1 \rightarrow \mathrm{LUMO}, \\
\mathrm{HOMO}-2 \rightarrow \mathrm{LUMO}+1\end{array}$ \\
\hline 3 & 4.0864 & 303.4069 & $\begin{array}{l}\mathrm{HOMO}-1 \rightarrow \mathrm{LUMO}+1, \\
\mathrm{HOMO}-2 \rightarrow \mathrm{LUMO}\end{array}$ \\
\hline 4 & 4.6548 & 266.3577 & $\mathrm{n} / \mathrm{a}$ \\
\hline 5 & 4.6551 & 266.3406 & $\mathrm{n} / \mathrm{a}$ \\
\hline
\end{tabular}

The lowest excitation occurs at a wavelength of $307.66 \mathrm{~nm}$. We therefore adopted this wavelength in the calculation of the full-resonance ROA spectrum. However, we can also see from Table 1 that the second excitation occurs at almost the same energy. Hence, both states will simultaneously be involved in resonance effects. As we will understand below, this fact leads to very interesting effects in the ROA spectra. Moreover, as the third lowest state (at $303.41 \mathrm{~nm}$ above the ground state) is also very close to the aforementioned two states, it is likely that also this state will usually be involved in resonance effects, albeit to a somewhat lesser extent. Resonance effects with states beyond the third lowest excited state will be negligible, since the excitation wavelengths are at least $50 \mathrm{~nm}$ larger compared to the lowest excitation (this is also indicated by the findings of Ref. 24).

It is also instructive to analyze the molecular orbitals (MOs) involved in the three lowest electronic excitations. The dominant MO transitions involved in these excitations are also listed in Table 1 and the respective MOs are shown in Fig. 2. The first excitation is mostly a transition from the highest occupied MO (HOMO) to the lowest unoccupied MO (LUMO). While the HOMO is localized at the metal center, the LUMO is more extended, covering also four of the nitrogen atoms. One can therefore interpret this transition as moving some electronic charge from the rhodium atom to the nitrogen atoms. This interpretation remains valid when considering also the other two dominating transitions, namely the transitions between $\mathrm{HOMO}-1$ and $\mathrm{LUMO}+1$, and between HOMO -2 and LUMO. HOMO -2 and 
HOMO -1 are mostly localized at the central rhodium atom, while LUMO +1 is, like the LUMO, more extended, also covering several nitrogen atoms. Following the same argumentation, we find that also the second and third lowest transition represent a movement of charge from the metal center to the adjacent nitrogen atoms.

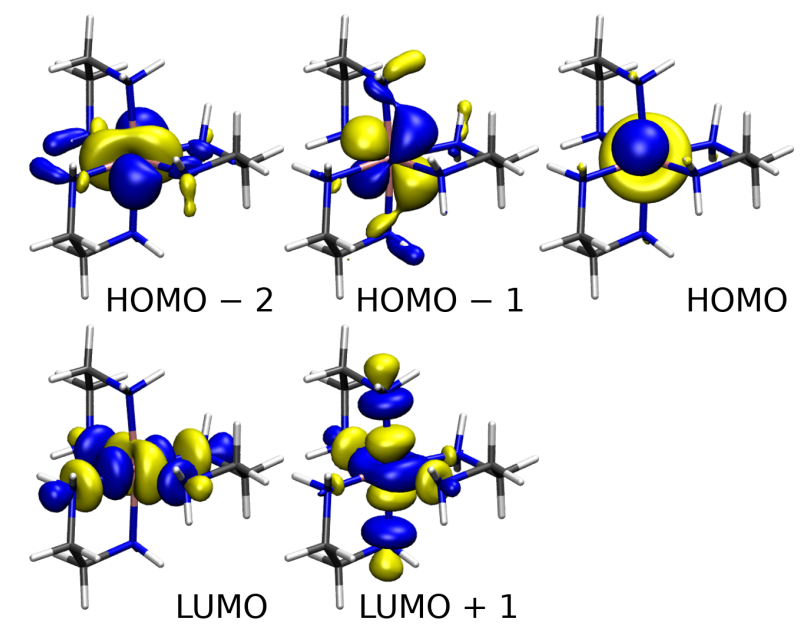

Figure 2: Molecular orbitals of $\left[\mathrm{Rh}(\mathrm{en})_{3}\right]^{3+}$ involved in the lowest three electronic excitations.

\subsection{ROA Spectra}

After having analyzed the electronic excitations playing a role in the (resonance) ROA spectra, we are now in a position to investigate these spectra. 


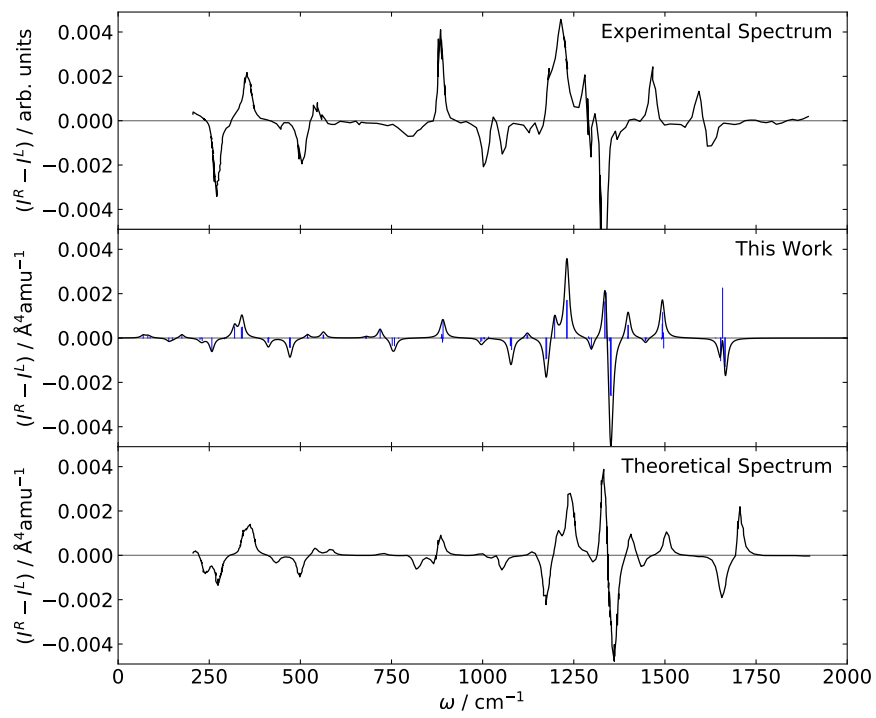

Figure 3: Comparison of the experimental (top panel) and theoretical (bottom panel) offresonance spectra of $\left[\mathrm{Rh}(\mathrm{en})_{3}\right]^{3+}$ as obtained by Humbert-Droz et al. ${ }^{19}$ to the off-resonance spectrum obtained in this work (middle panel).

First, we compare our results to the ones obtained by Humbert-Droz et al. ${ }^{19}$ In Fig. 3, we show both the experimental as well as the theoretical off-resonance spectrum obtained by these authors and compare it to the off-resonance spectrum obtained in this work using an excitation wavelength of $800 \mathrm{~nm}$. In the work by Humbert-Droz et al., ${ }^{19}$ an excitation wavelength of $532 \mathrm{~nm}$ was adopted. As we have elaborated in the previous section, this wavelength is in fact far away from any electronic excitations. We will see below that for wavelengths longer than $350 \mathrm{~nm}$, resonance effects do not play a major role anymore in the ROA spectrum of $\left[\mathrm{Rh}(\mathrm{en})_{3}\right]^{3+}$. The experimental spectrum is, therefore, effectively an off-resonance spectrum and it is possible to compare the spectra shown in Fig. 3 to each other, even though they have been obtained with different excitation wavelengths. The apparent ruggedness of the two spectra taken from Ref. 19 is in part due to the fact that it was extracted directly from the paper by Humbert-Droz et al. ${ }^{19}$ which introduced minor artifacts. Also note that Humbert-Droz et al. adopted a different computational methodology; most notably, instead of the PBE0 density functional, they used the B3LYP ${ }^{43-45}$ exchange-correlation functional (as implemented in Gaussian ${ }^{46}$ ). Therefore, some differences between the theoretical spectra are to be expected.

As we can see, the three spectra agree well with each other, in particular the two theoretical spectra. Below $1000 \mathrm{~cm}^{-1}$, the only notable differences between these 
two spectra are slightly different band shapes in the region between $200 \mathrm{~cm}^{-1}$ and $300 \mathrm{~cm}^{-1}$, and the $+/-$ couplet at about $750 \mathrm{~cm}^{-1}$, which is visible in the spectrum calculated by us, but not in the theoretical spectrum obtained by Humbert-Droz et $a l .{ }^{19}$ Instead of such a couplet, however, we find two weakly negative peaks at about $800 \mathrm{~cm}^{-1}$ which cannot be identified in our spectrum. Also above $1000 \mathrm{~cm}^{-1}$, the two theoretical spectra agree well. The only difference is in the region between $1650 \mathrm{~cm}^{-1}$ and $1750 \mathrm{~cm}^{-1}$. Here, we find two negative bands in our spectrum, while there is a rather strong $-/+$ couplet in the spectrum reported in Ref. 19 (note that in the experimental spectrum, there is a $+/-$ couplet in this spectral range). However, also in the spectrum obtained in our study, we find a normal mode with a strongly positive ROA intensity in this wavenumber range. However, it is completely covered up by the near-lying modes which are all associated with negative ROA intensity. If this mode would be shifted to slightly larger wavenumbers, also our spectrum would feature a $-/+$ couplet. This highlights the fact that the overall band shape obtained in ROA spectra is highly sensitive to such comparatively small changes ( $c f$., also Ref. 47). Therefore, in all ROA spectra reported in this study, we will always also show the individual normal modes.

At wavenumbers below $1000 \mathrm{~cm}^{-1}$, the experimentally measured intensities are clearly larger than the calculated ones, but the wavenumbers of the measured peaks matches those of the calculated peaks almost perfectly. The $+/-$ couplet at about $750 \mathrm{~cm}^{-1}$, which we find in our spectrum, cannot be identified in the experimental spectrum. Instead, the experimental spectrum features a broad, negative band at around $800 \mathrm{~cm}^{-1}$. This is consistent with what we find in the theoretical spectrum obtained by Humbert-Droz et al. ${ }^{19}$ At wavenumbers above $1000 \mathrm{~cm}^{-1}$, the relative intensity ratio between the two strongly positive peaks at about $1200 \mathrm{~cm}^{-1}$ and $1300 \mathrm{~cm}^{-1}$, respectively, is correct reproduced in our spectrum, while it is reversed in the spectrum given in Ref. 19. However, these are minor differences, and the overall agreement of all three spectra is very satisfactory.

Now, we can analyze all our ROA spectra and how they are affected by resonances with electronic states. The individual spectra are shown in Figs. 4-6. The fullresonance spectrum (at $307.66 \mathrm{~nm}$, see top spectrum in Fig. 4) shows bisignate peaks, i.e., it is not monosignate, as is often the case for RROA spectra. This is because RROA spectra are only monosignate in the case of resonance with exactly one excited electronic state. In our case, however, we have seen above that the three lowest electronic excitations are so close to each other in energy that they all contribute to the resonance effects observed. It is well known that in such situations, bisignate RROA spectra can result. ${ }^{22-24}$ The intensity of the full-resonance spectrum reaches absolute values of up to $0.12 \AA^{4} \mathrm{amu}^{-1}\left(\right.$ at $\left.520 \mathrm{~cm}^{-1}\right)$. This is larger than the maximum intensity in the case of the spectrum recorded at $800 \mathrm{~nm}$ (which 
is a pure off-resonance spectrum) where the maximum absolute intensity reaches only about $0.005 \AA^{4} \mathrm{amu}^{-1}$. We therefore clearly see an enhancement of the ROA intensity through the resonance with electronically excited states, as is expected.

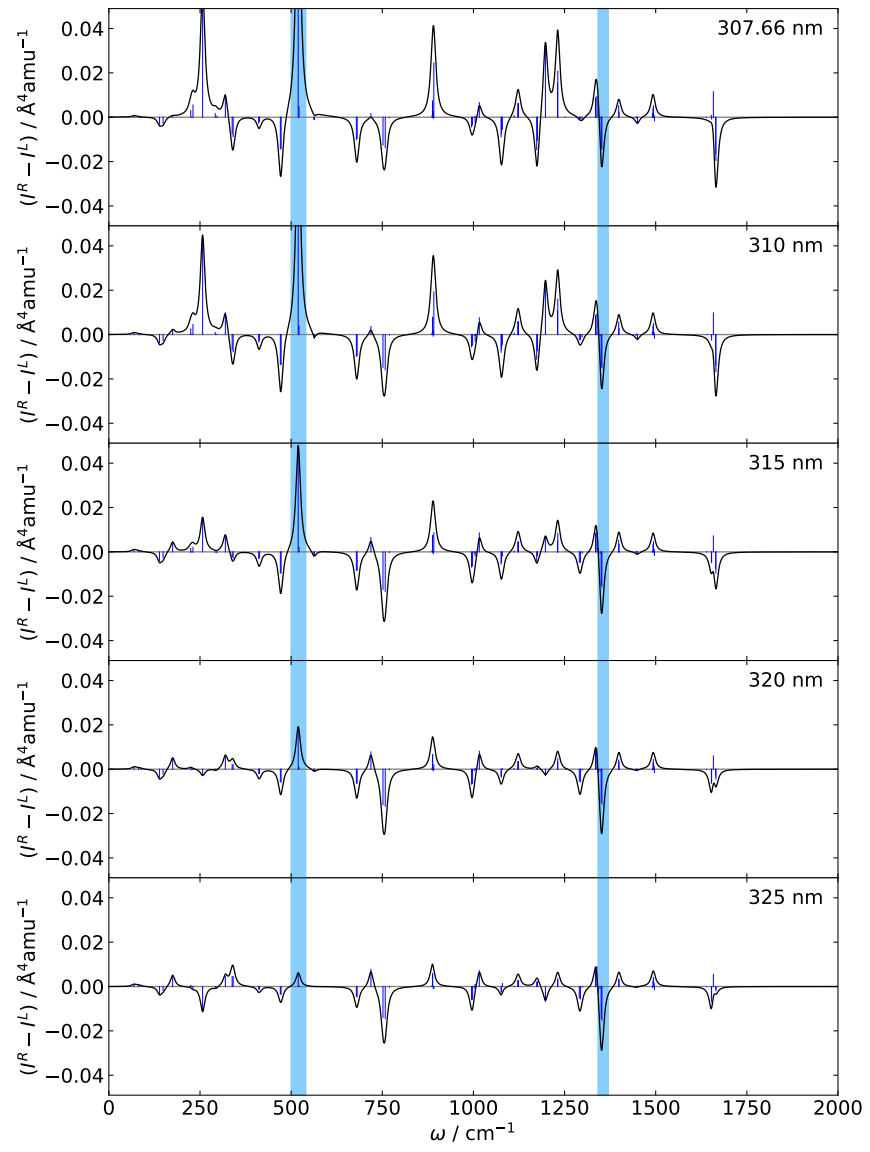

Figure 4: ROA spectra for five different wavelengths at or close to resonance with the first three excited electronic states (in descending order from top to bottom). The two bands primarily analyzed in this study are highlighted.

Increasing the excitation wavelength from $307.66 \mathrm{~nm}$ to $310 \mathrm{~nm}$ yields a spectrum which looks very similar to the full-resonance spectrum (see the second spectrum from above in Fig. 4). All band shapes and relative intensity differences are basically the same, but the overall intensity is reduced, reaching a maximum value of about $0.095 \AA^{4} \mathrm{amu}^{-1}$ at $520 \mathrm{~cm}^{-1}$. Increasing the excitation wavelength to $315 \mathrm{~nm}$, we find that the intensity is further reduced; the maximum value is now roughly $0.048 \AA^{4} \mathrm{amu}^{-1}$ (still at $520 \mathrm{~cm}^{-1}$ ). Relative intensity differences are now strong enough to have a visible influence on the overall band shapes. For example, the 
band at $1660 \mathrm{~cm}^{-1}$ does no longer appear as a single sharp peak, but is split into two peaks.

In the ROA spectrum at $320 \mathrm{~nm}$, the maximum intensity is no longer found for the band at $520 \mathrm{~cm}^{-1}$ but for the one at about $760 \mathrm{~cm}^{-1}$. Its absolute intensity (about $\left.0.03 \AA^{4} \mathrm{amu}^{-1}\right)$ is roughly as large as the one for the peak at about $1350 \mathrm{~cm}^{-1}$. When compared to the full-resonance spectrum at $307.66 \mathrm{~nm}$, the overall spectrum has changed significantly. While certain spectral regions (e.g., between $1400 \mathrm{~cm}^{-1}$ and $1500 \mathrm{~cm}^{-1}$ ) are almost the same in both spectra, other regions (e.g., between $200 \mathrm{~cm}^{-1}$ and $300 \mathrm{~cm}^{-1}$ ) differ significantly. In the case of the full-resonance spectrum, we see a strongly positive band between $200 \mathrm{~cm}^{-1}$ and $300 \mathrm{~cm}^{-1}$. The spectrum at $320 \mathrm{~nm}$ features a very weak positive peak and a slightly stronger, negative band in the same spectral region. Also, at about $700 \mathrm{~cm}^{-1}$, we can identify a positive band in the spectrum at $320 \mathrm{~nm}$. This was completely masked in the full-resonance spectrum (since the corresponding normal mode has a much weaker, albeit also positive, intensity in the full-resonance case). Furthermore, also between $1200 \mathrm{~cm}^{-1}$ and $1300 \mathrm{~cm}^{-1}$ the two spectra differ strongly. In the full-resonance case, we find here two strongly positive peaks, followed by a weaker negative one. At $320 \mathrm{~nm}$, we find only one positive peak, much less intense, while the negative peak has gained intensity. The normal mode at $1252 \mathrm{~cm}^{-1}$, causing the second strongly positive peak in the spectrum at $307.66 \mathrm{~nm}$, has almost zero ROA intensity at $320 \mathrm{~nm}$, which is why it can no longer be clearly identified in the corresponding spectrum. The spectrum with an excitation wavelength of $325 \mathrm{~nm}$ looks very similar to the one at $320 \mathrm{~nm}$, but we can still find a few distinct changes. For example, the most intense peak is now found at about $1350 \mathrm{~cm}^{-1}$. The band at about $520 \mathrm{~cm}^{-1}$ has further lost intensity.

At an excitation wavelength of $330 \mathrm{~nm}$, the overall intensity is further reduced ( $c f$., Fig. 5). We can still find some significant changes in the overall band shape. In particular, the positive band at $520 \mathrm{~cm}^{-1}$ has almost completely vanished at $330 \mathrm{~nm}$. At an excitation wavelength of $350 \mathrm{~nm}$, one can see that the overall intensity continues to be reduced. Compared to the spectrum at $330 \mathrm{~nm}$, however, the overall band shape is conserved. In all spectra with excitation wavelengths larger than $350 \mathrm{~nm}$, we find well-conserved band shapes. Therefore, one can say that already at $350 \mathrm{~nm}$, the shape of the RROA spectrum is identical to the one of a pure off-resonance spectrum at $800 \mathrm{~nm}$. However, the overall intensity is continuously decreasing with increasing excitation wavelength. This might be due to very weak resonance effects (absorption tail). 


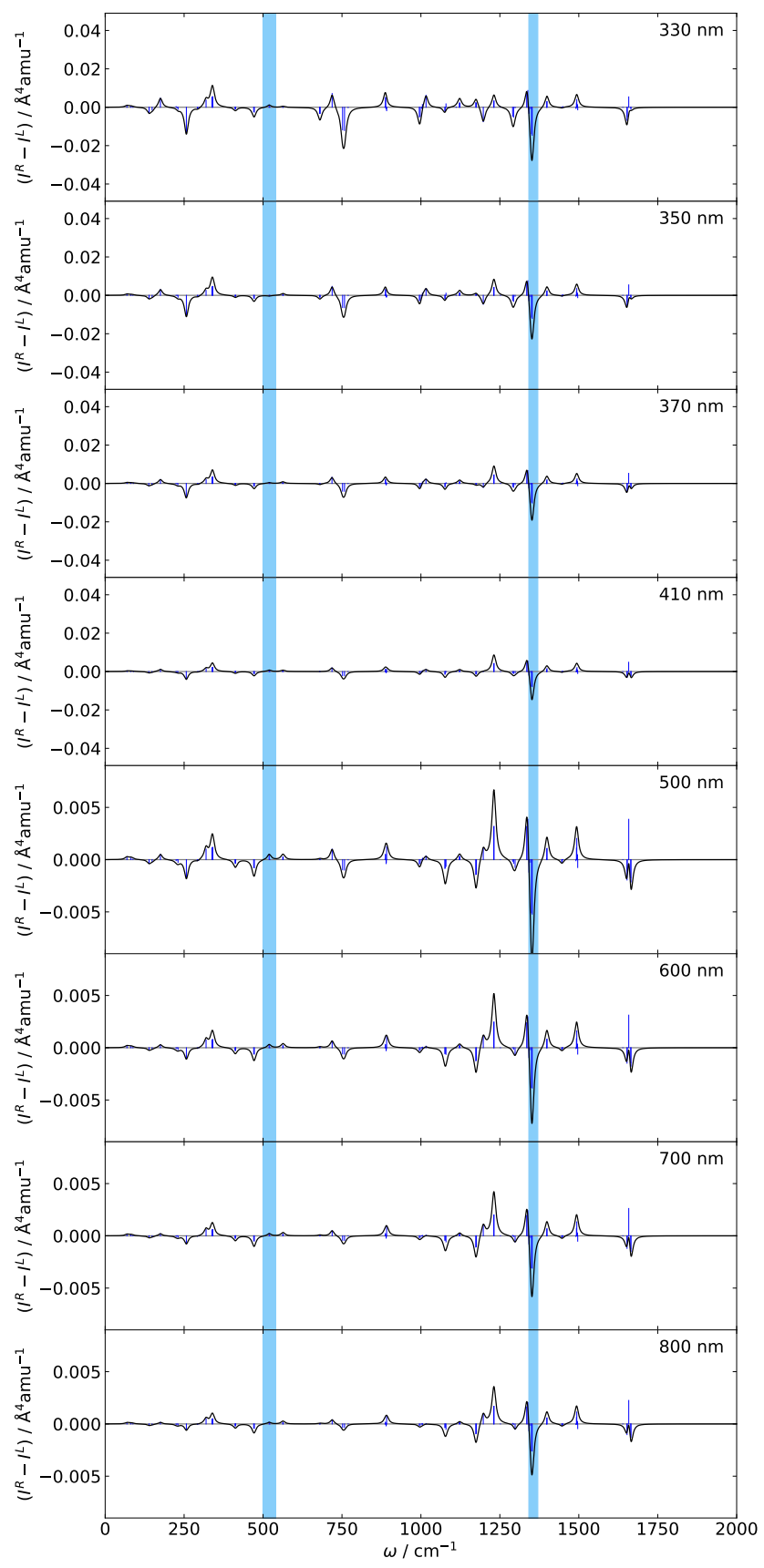

Figure 5: ROA spectra for different off-resonant wavelengths. Note that for wavelengths larger than $410 \mathrm{~nm}$, a smaller intensity range is plotted for the sake of clarity. The two bands primarily analyzed in this study are highlighted. 
An interesting observation can be made for the normal mode at around $720 \mathrm{~cm}^{-1}$. We find that the intensity of this mode increases when moving away from resonance, until the highest intensity is observed at $320 \mathrm{~nm}$. For larger wavelengths, the intensity decreases again. When examining the intensities of the individual normal modes closer, we find that such a behavior is found for many of the normal modes (see Supporting Information). The reason for this intricate behavior is not fully clear. However, it is known that resonance with multiple excited states can lead to de-enhancement effects and concomitantly complex line shapes. ${ }^{24}$ We could hypothesize that the third excited electronic state has a de-enhancing effect on the ROA spectrum. At longer wavelengths, the third excited state (303 nm excitation wavelength) is less important than the first two, and concomitantly its de-enhancing effect should be diminished. It is, therefore, interesting to see how the spectrum looks like at shorter wavelengths, when this third state should be more important.

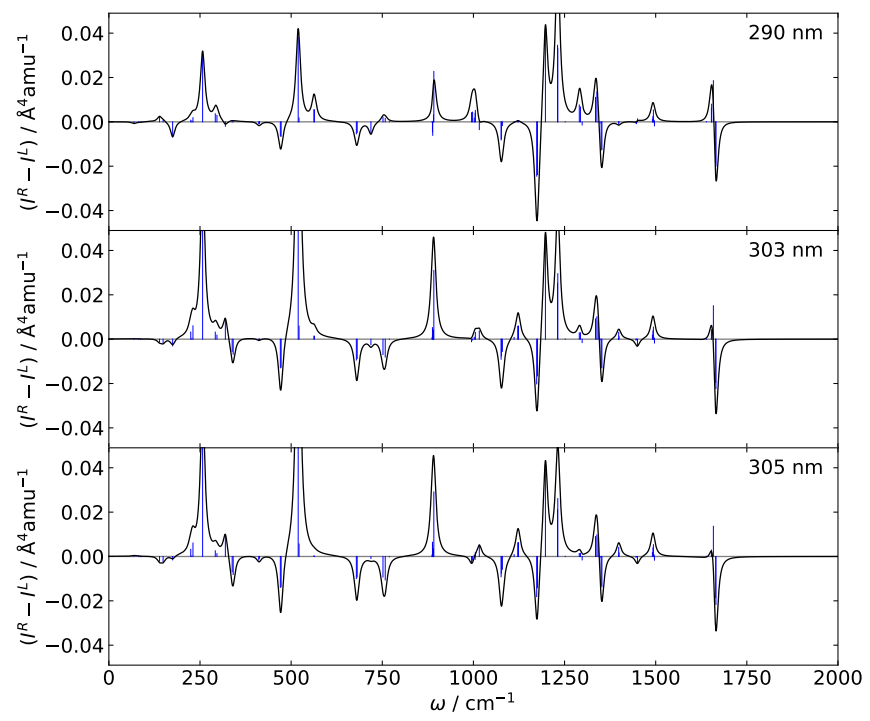

Figure 6: ROA spectra for three pre-resonance wavelengths.

Hence, we also calculated spectra at $305 \mathrm{~nm}, 303 \mathrm{~nm}$, and $290 \mathrm{~nm}$ (see Fig. 6). Overall, all these spectra have a rather large resemblance to the spectrum at full resonance, but it can be argued (based on visual inspection), that the spectrum at $325 \mathrm{~nm}$ is more similar to the spectrum at $307.66 \mathrm{~nm}$ than the spectrum at $290 \mathrm{~nm}$ (note that both off-resonant wavelengths have roughly the same distance to the resonant wavelength). Therefore, we might conclude that, as expected, the third excited state contributes much more to the spectrum at $290 \mathrm{~nm}$ and therefore leads to bigger changes. Even though the spectrum at $305 \mathrm{~nm}$ looks very similar to the spectrum 
at full resonance, some distinct differences can be identified. Most prominently, we can identify a weakly positive band at around $1650 \mathrm{~cm}^{-1}$, which is not visible at full resonance (although also in this case, there are normal modes in this spectral region associated with positive ROA intensity). This positive band is further accentuated at smaller wavelengths, and at $290 \mathrm{~nm}$ we find a strong $+/-$ couplet in this region. When comparing the spectrum at $290 \mathrm{~nm}$ to the one at $307.66 \mathrm{~nm}$, we find many differences. While many bands have decreased in intensity (as one would expect based on our hypothesis of a de-enhancing effect of the third lowest electronic excitation), there are also many peaks which grow in intensity. For example, the negative peak at around $1180 \mathrm{~cm}^{-1}$ and the two positive peaks between $1200 \mathrm{~cm}^{-1}$ and $1250 \mathrm{~cm}^{-1}$. Hence, the third excited state has not always de-enhancing effects on all normal modes, but the relation appears to be more complicated.

We have seen that the band at $520 \mathrm{~cm}^{-1}$ changes significantly when increasing the excitation wavelength; starting as the strongest peak in the entire spectrum, it becomes continuously weaker until it vanishes at $350 \mathrm{~nm}$. Moreover, the negative peak at about $1350 \mathrm{~cm}^{-1}$ is more or less unaffected. It is interesting, therefore, to investigate these two peaks more closely. An analysis in terms of so-called group coupling matrices (GCMs) as proposed by $\mathrm{Hug}^{48}$ provides an appealing means of assigning the total ROA intensity of a given normal mode to parts of a molecule. For such an analysis, the proper choice of these parts is crucial. In our case, we adopt the rather fine-grained partitioning of $\left[\mathrm{Rh}(\mathrm{en})_{3}\right]^{3+}$ as shown in Fig. 7; note that also Humbert-Droz et al. have found this partitioning to be useful for some of the normal modes they analyzed. ${ }^{19}$

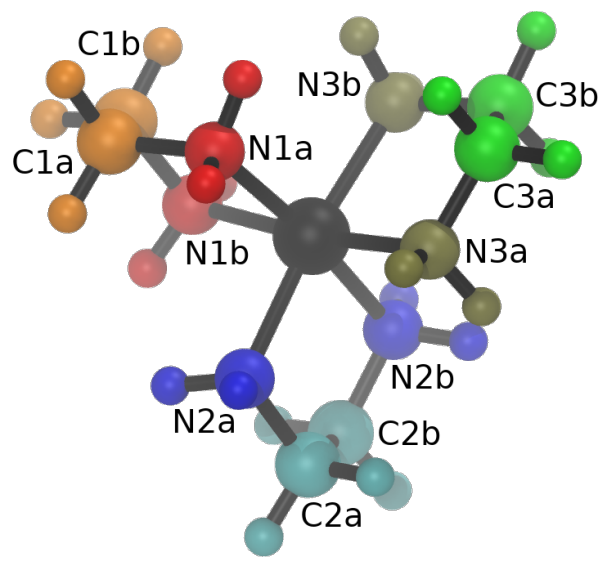

Figure 7: Groups of $\left[\mathrm{Rh}(\mathrm{en})_{3}\right]^{3+}$ adopted for all group coupling matrices. 

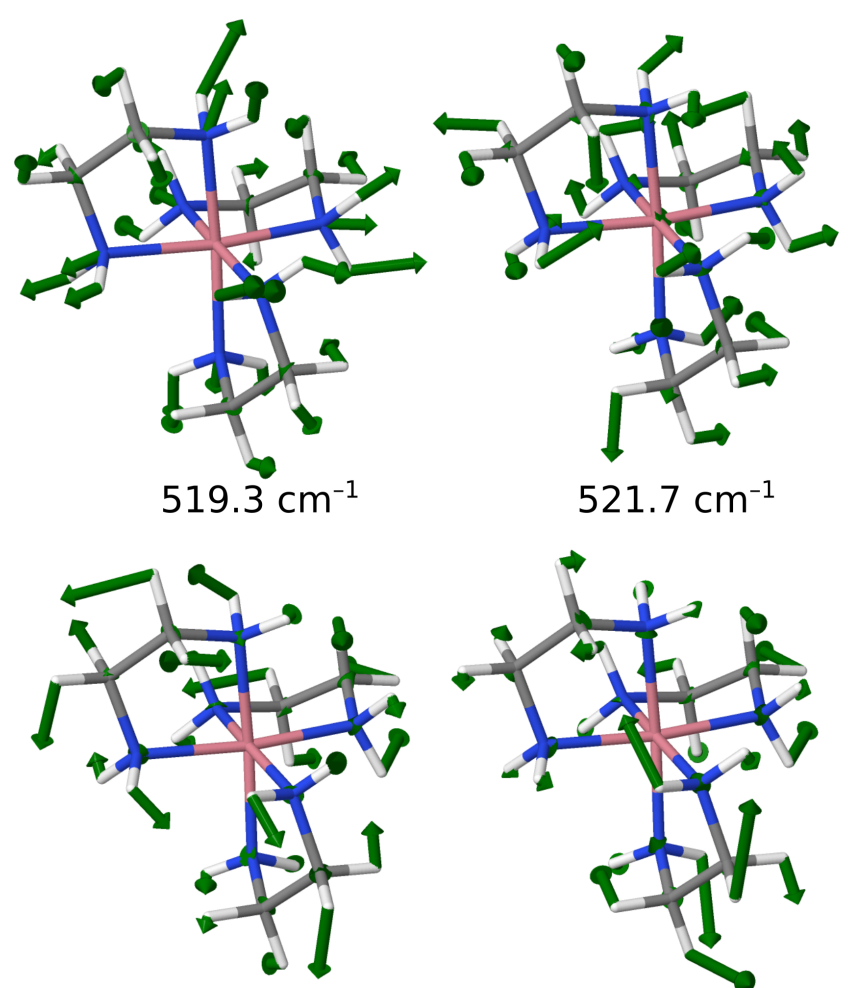

$1347.3 \mathrm{~cm}^{-1}$

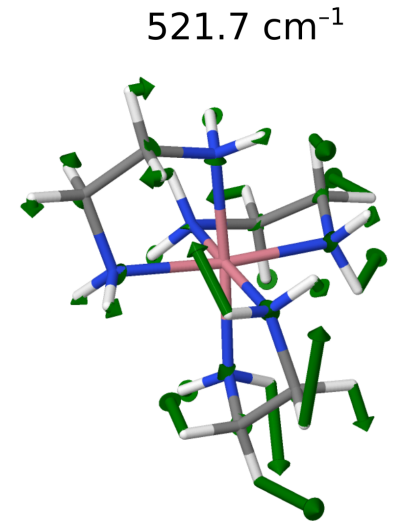

$1350.4 \mathrm{~cm}^{-1}$

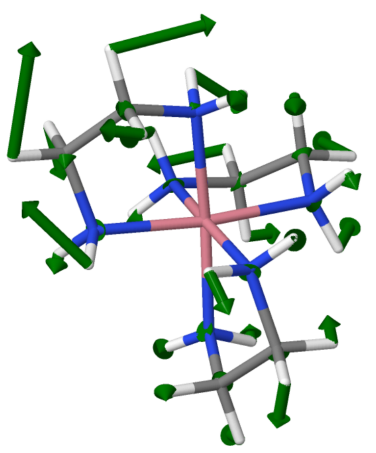

$1352.5 \mathrm{~cm}^{-1}$

Figure 8: Normal modes selected for closer analysis. The wavenumber of each normal mode is given below its representation.

The band at about $520 \mathrm{~cm}^{-1}$ is composed of two normal modes. The first normal mode (at $519.3 \mathrm{~cm}^{-1}$ ) represents a symmetric stretching vibration of all six $\mathrm{Rh}-\mathrm{N}$ bonds, while the second mode (at $521.7 \mathrm{~cm}^{-1}$ ) describes a more complicated torsional motion of the three ligands (see Fig. 8). To a good approximation, we can neglect the contribution of this second mode to the positive band at around $520 \mathrm{~cm}^{-1}$, since its intensity is always about an order of magnitude smaller than the one of the first mode. We are therefore left with the analysis of the mode at $519.3 \mathrm{~cm}^{-1}$. Its GCMs for different excitation wavelengths are shown in Fig. 9. 
$307.66 \mathrm{~nm}$

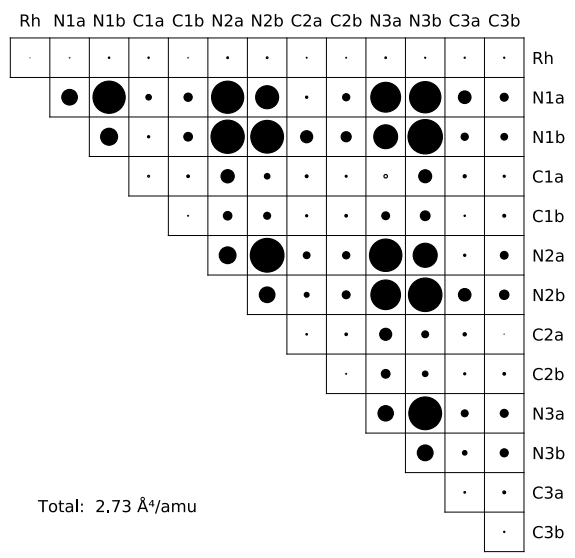

$320 \mathrm{~nm}$

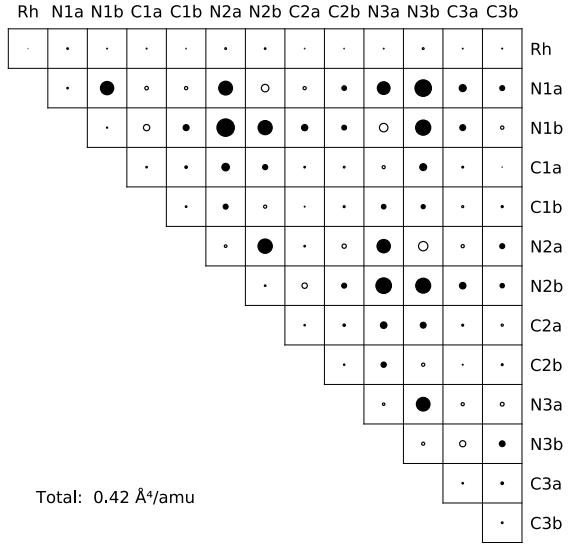

$315 \mathrm{~nm}$

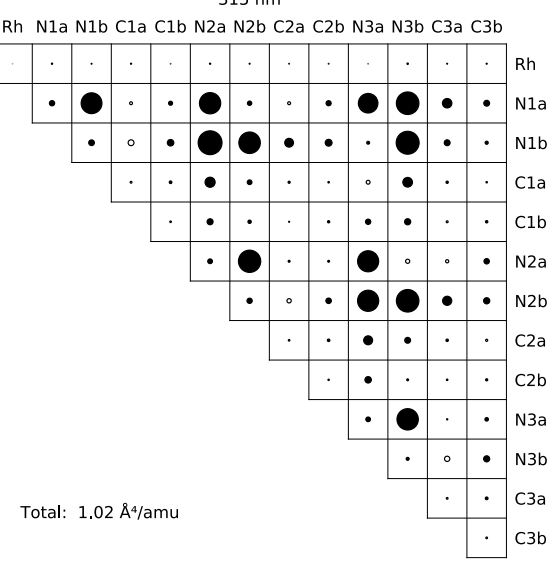

$350 \mathrm{~nm}$

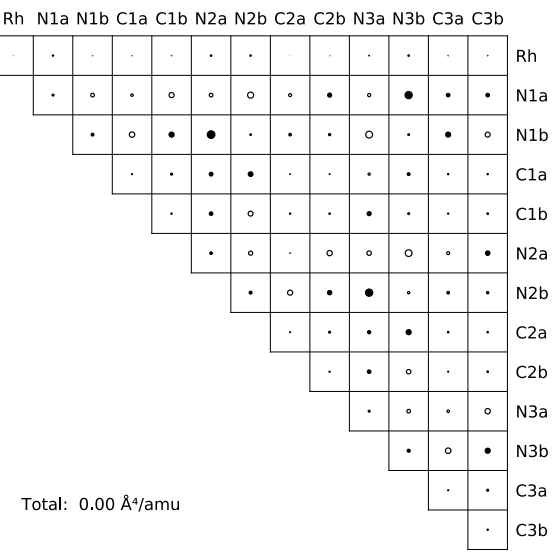

Figure 9: Group coupling matrices for the normal mode at $519.3 \mathrm{~cm}^{-1}$ for four different excitation wavelengths (top left: $307.66 \mathrm{~nm}$; top right: $315 \mathrm{~nm}$; bottom left: $320 \mathrm{~nm}$; bottom right: $350 \mathrm{~nm}$ ). Positive contributions are represented by filled circles, while negative contributions are drawn as empty circles. The radius of the circle is proportional to the size of the corresponding GCM matrix element.

As we can see, at $307.66 \mathrm{~nm}$ almost all parts of the molecule contribute to the positive ROA intensity. Only the coupling between the $\mathrm{CH}_{2}$ group $\mathrm{Cla}$ and $\mathrm{NH}_{2}$ group N3a leads to weakly negative ROA intensity, but this can safely be neglected. The biggest contributions to the overall ROA intensity are clearly given by the six $\mathrm{NH}_{2}$ groups as well as couplings between them. In fact, these couplings lead to stronger contributions than the individual $\mathrm{NH}_{2}$ groups provide, and couplings between adjacent $\mathrm{NH}_{2}$ groups are not stronger than couplings between distant $\mathrm{NH}_{2}$ groups, but not all couplings are equally strong: The coupling of N1a and N2b, N1b and N3a, and N2a and N3b are clearly weaker than the other couplings. From Fig. 7, 
we see that these combinations of $\mathrm{NH}_{2}$ groups are always forming an $\mathrm{N}-\mathrm{Rh}-\mathrm{N}$ angle of about $180^{\circ}$, while for any other two $\mathrm{NH}_{2}$ groups, the $\mathrm{N}-\mathrm{Rh}-\mathrm{N}$ angle is about $90^{\circ}$. Hence, we conclude that the weaker couplings must be due to this specific spatial arrangement.

For the larger excitation wavelength of $315 \mathrm{~nm}$, these strong contributions have become significantly weaker, but they still play the dominant role. In particular, the "weak" couplings between $\mathrm{NH}_{2}$ groups (i.e., the couplings between N1a and $\mathrm{N} 2 \mathrm{~b}$, between N1b and N3a, and between N2b and N3a) are greatly reduced, being now not more important than any of the other couplings. Several couplings leading to negative ROA intensity are visible now, most notably the coupling between N2a and N3b (which contributed much positive ROA intensity at full resonance).

At $320 \mathrm{~nm}$, the couplings between the $\mathrm{NH}_{2}$ groups are further reduced, and the intensity which the individual $\mathrm{NH}_{2}$ groups provide directly (i.e., not through couplings) can be safely neglected. All weak $\mathrm{NH}_{2}$ couplings are now clearly negative; in fact, they are the most negative contributions of the entire GCM. Together with the remaining, weaker negative couplings, they can almost completely cancel out all positive contributions.

Finally, at $350 \mathrm{~nm}$, no dominating parts can be clearly identified in the GCM. Positive and negative contributions cancel each other exactly. At even larger excitation wavelengths, the situation remains essentially unchanged, and the overall intensity is always zero. In summary, we have seen that the strongly positive ROA intensity under resonance conditions originates from the $\mathrm{NH}_{2}$ groups. These contributions quickly vanish when proceeding to larger excitation wavelengths, concomitantly reducing the total intensity of the corresponding normal mode.

The negative band at about $1350 \mathrm{~cm}^{-1}$ results from three normal modes, at $1347.3 \mathrm{~cm}^{-1}$, at $1350.4 \mathrm{~cm}^{-1}$, and at $1352.5 \mathrm{~cm}^{-1}$. All three modes represent a twisting motion of the $\mathrm{NH}_{2}$ and $\mathrm{CH}_{2}$ groups ( $c f$. ., Fig. 8). For the negative band around $1350 \mathrm{~cm}^{-1}$, the mode at $1347.3 \mathrm{~cm}^{-1}$ can be neglected to a first approximation, as its intensity is never larger than $0.02 \AA^{4} \mathrm{amu}^{-1}$, while the other two modes have intensities around $0.36 \AA^{4} \mathrm{amu}^{-1}$ under resonance conditions. For the mode at $1350.4 \mathrm{~cm}^{-1}$, the origin of the total ROA intensity is difficult to identify (see Fig. 10). Under resonance conditions, many couplings have roughly the same total strength. However, one can say that the most dominant contributions originate from the parts $\mathrm{N} 2 \mathrm{~b}-\mathrm{C} 3 \mathrm{~b}$, and couplings of these parts with other parts of the molecule. When going to $315 \mathrm{~nm}$, this general pattern remains unaffected, but many of the couplings are reduced in absolute value. However, the positive couplings appear to be reduced more than the negative couplings; hence, the total intensity even decreases to a certain degree. At $320 \mathrm{~nm}$, almost all positive couplings between parts of N2b-C3b have vanished. 
At even larger wavelengths, the same general trend is observed: while the coupling pattern remains largely unaffected, the absolute intensities of all couplings diminish somewhat. Hence, the total intensity of this mode grows continuously weaker (albeit this decrease is by far not as strong as in the case of the band at around $520 \mathrm{~cm}^{-1}$ ). For the third mode of this band (at $1352.5 \mathrm{~cm}^{-1}$ ), the same general trend is valid ( $c f .$, Fig. 11). The only difference is that under resonance conditions, most of the dominant coupling elements are among parts N1a-C1b.

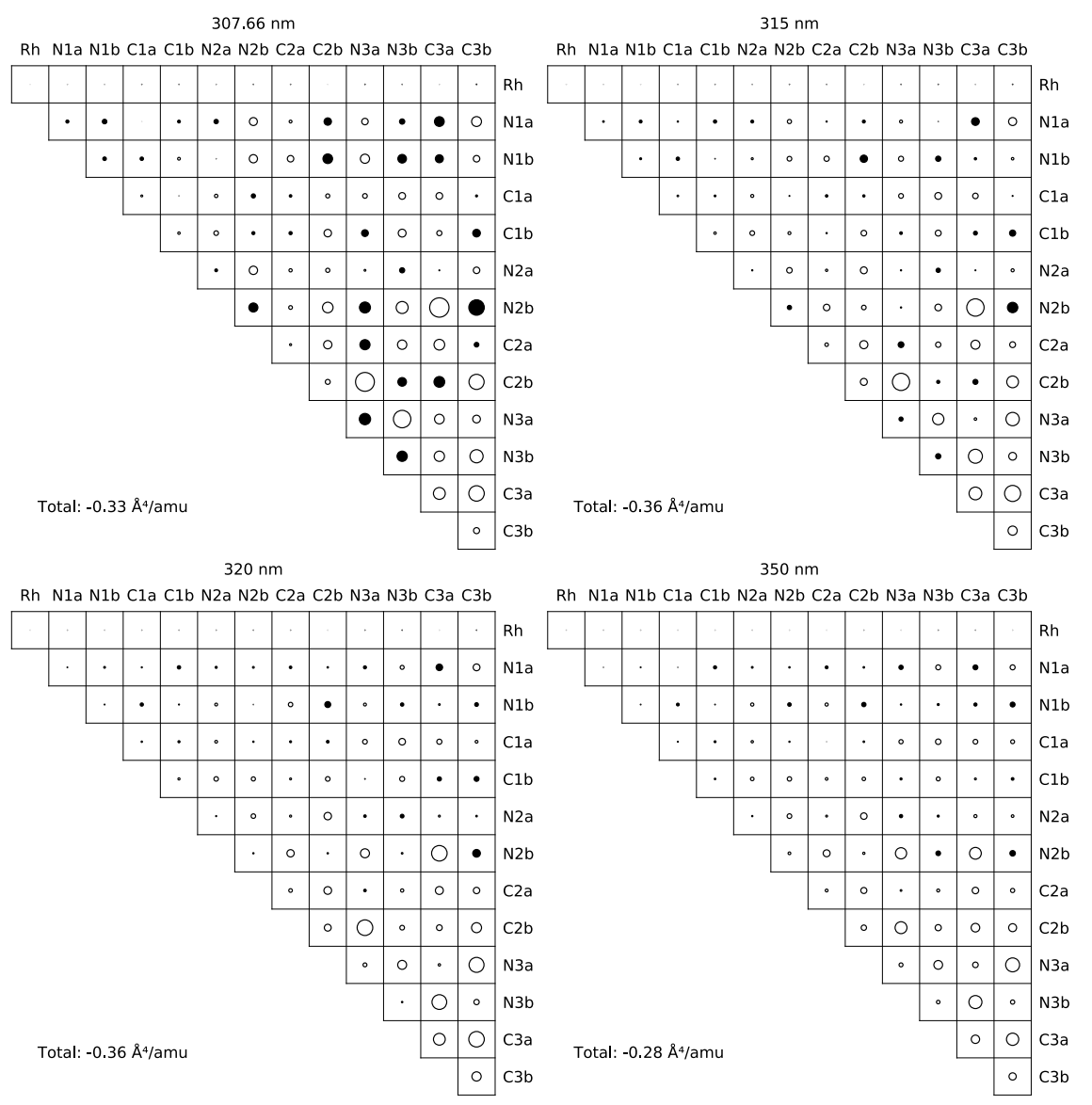

Figure 10: Group coupling matrices for the normal mode at $1350.4 \mathrm{~cm}^{-1}$ for four different excitation wavelengths (top left: $307.66 \mathrm{~nm}$; top right: $315 \mathrm{~nm}$; bottom left: $320 \mathrm{~nm}$; bottom right: $350 \mathrm{~nm}$ ). Positive contributions are represented by filled circles, while negative contributions are drawn as empty circles. The radius of the circle is proportional to the size of the corresponding GCM matrix element. 


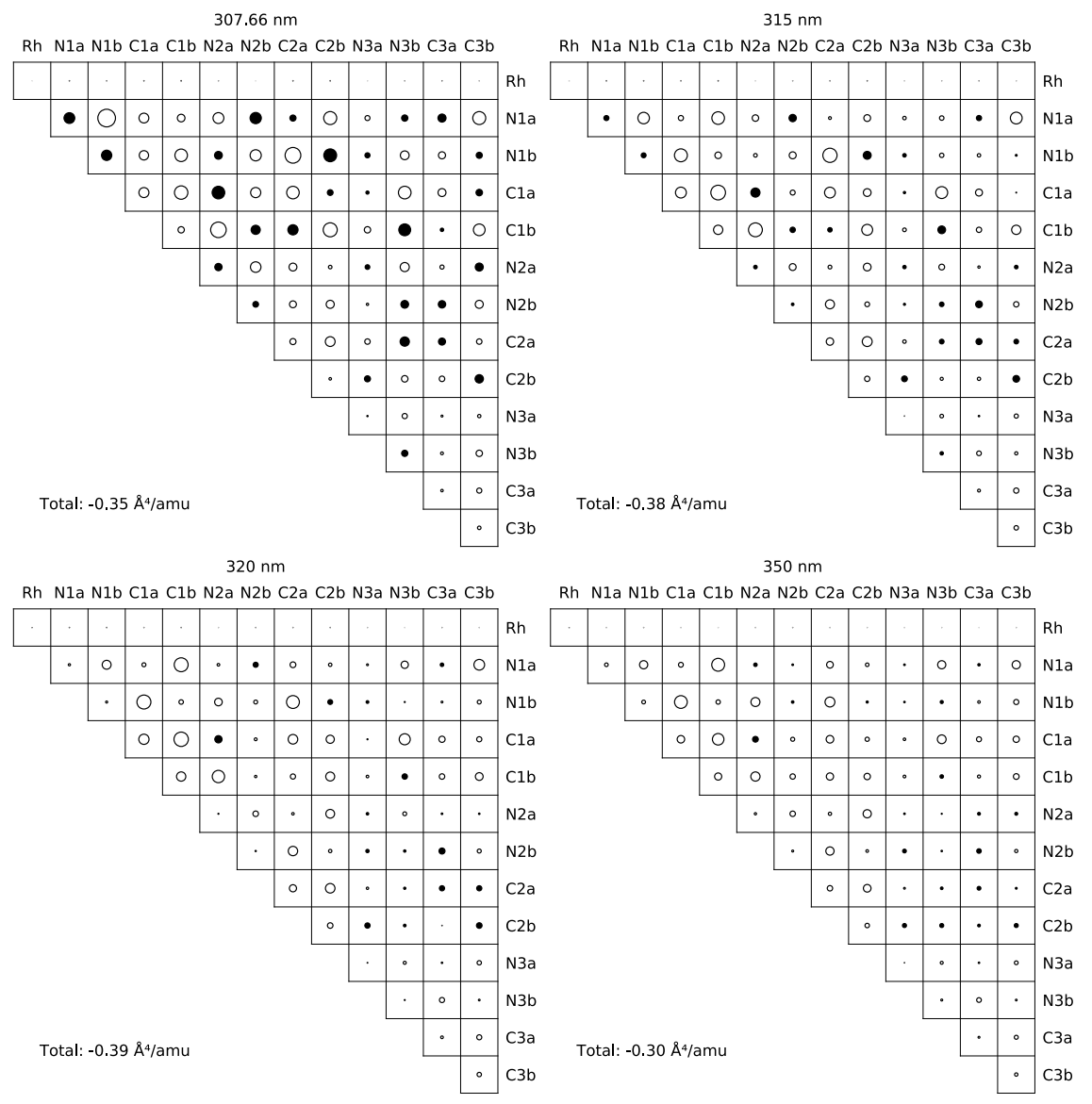

Figure 11: Group coupling matrices for the normal mode at $1352.5 \mathrm{~cm}^{-1}$ for four different excitation wavelengths (top left: $307.66 \mathrm{~nm}$; top right: $315 \mathrm{~nm}$; bottom left: $320 \mathrm{~nm}$; bottom right: $350 \mathrm{~nm}$ ). Positive contributions are represented by filled circles, while negative contributions are drawn as empty circles. The radius of the circle is proportional to the size of the corresponding GCM matrix element.

Inspecting the GCMs shown in Figs. 9, 10, and 11, we also see that the rhodium atom never contributes significantly to the observed intensities, neither directly, nor through couplings with any of the other groups. We note that Humbert-Droz et $a l$. have found the same result for the bands they analyzed in greater detail, ${ }^{19}$ albeit only in the off-resonant case.

When analyzing the electronic excitations involved in the ROA spectra (see above), we find that electronic charge is moved from the metal center towards the $\mathrm{NH}_{2}$ groups. It is therefore not too surprising that in the band around $520 \mathrm{~cm}^{-1}$ (which changed significantly when approaching larger excitation wavelengths), most of the 
intensity under resonance conditions originates from exactly these $\mathrm{NH}_{2}$ groups. We may hypothesize that any normal mode is particularly affected by resonance effects if the $\mathrm{NH}_{2}$ groups are involved to a large extent. In order to elaborate on this, we calculated the amount to which these groups are involved in every normal mode. Furthermore, for every single normal mode, we identified the maximum as well as the minimum absolute intensity observed. The data for selected normal modes is shown in Table 2.

First of all, we see that the normal mode at $519.3 \mathrm{~cm}^{-1}$ features a rather large involvement of the $\mathrm{NH}_{2}$ groups. This would also be expected, since this normal mode essentially represents a stretching vibration of all six $\mathrm{Rh}-\mathrm{N}$ bonds. As already mentioned above, this mode features a large intensity under full resonance conditions (in fact, the highest intensity is even observed at a slightly shorter wavelength), and then collapses to a peak which is hardly identifiable at very large excitation wavelengths. Similarly, the almost constant intensity of the two normal modes at $1350.4 \mathrm{~cm}^{-1}$ and $1352.5 \mathrm{~cm}^{-1}$ can be clearly seen. Since these normal modes represent a twisting motion of both the $\mathrm{CH}_{2}$ and the $\mathrm{NH}_{2}$ groups, the latter groups have a much lower contribution to the total vibration.

Next, we study the two $\mathrm{CH}_{2}$ scissoring vibrations at $1494.2 \mathrm{~cm}^{-1}$ and $1496.6 \mathrm{~cm}^{-1}$, respectively. Both normal modes have very similar wavenumbers and describe basically identical vibrations, albeit of different $\mathrm{CH}_{2}$ groups. Naturally, the $\mathrm{NH}_{2}$ groups do almost not participate in either of the two normal modes. However, the first of these normal modes (at $1494.2 \mathrm{~cm}^{-1}$ ) is strongly enhanced when moving to larger excitation wavelengths, reaching a maximum at $370 \mathrm{~nm}$, while the other normal mode is much less affected and follows exactly the opposite trend, featuring the highest intensity at the shortest excitation wavelength. This intriguing fact can also be seen in the group coupling matrices of these two normal modes, shown in Fig. 12. Note that the coupling matrix of the first normal mode changes significantly and in a qualitative fashion between $350 \mathrm{~nm}$ and $370 \mathrm{~nm}$. For example, the coupling between the two $\mathrm{CH}_{2}$ groups C1a and C2b (cf., Fig. 7) is negative at $350 \mathrm{~nm}$, but it becomes strongly positive at $370 \mathrm{~nm}$. Likewise, the coupling between groups $\mathrm{C} 2 \mathrm{~b}$ and C3a changes from a negative contribution at $350 \mathrm{~nm}$ to a positive contribution at $370 \mathrm{~nm}$. Since these contributions are also rather large in magnitude (in fact, they are the largest couplings across the entire GCM), the total intensity of this normal mode is significantly increased. In contrast to this, the corresponding GCMs for the second normal mode are almost identical to each other. Furthermore, this example clearly shows that the amount of $\mathrm{NH}_{2}$ involvement in a given normal mode alone is not sufficient as a proxy for the prediction of how strongly this mode will be affected by resonance effects. Clearly, the first of these two modes is strongly affected by resonance effects, but the $\mathrm{NH}_{2}$ groups are almost not involved in its vibration. 


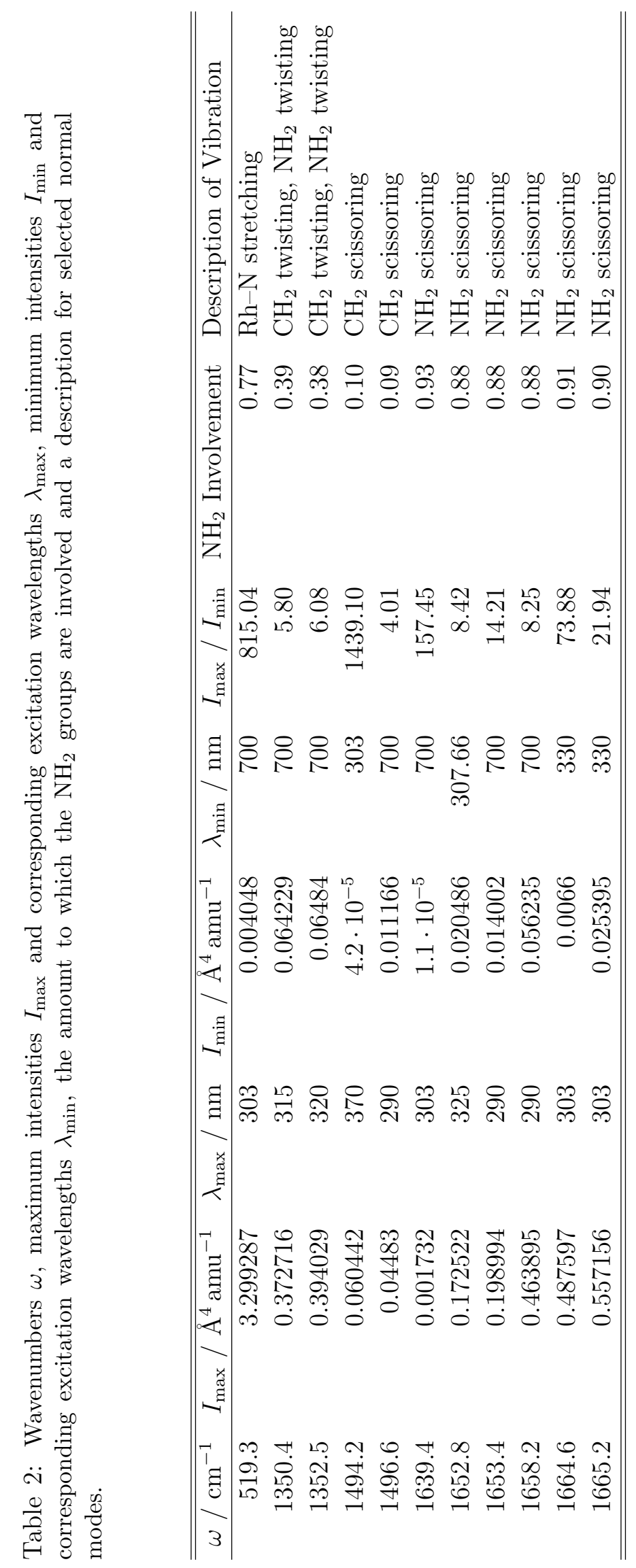




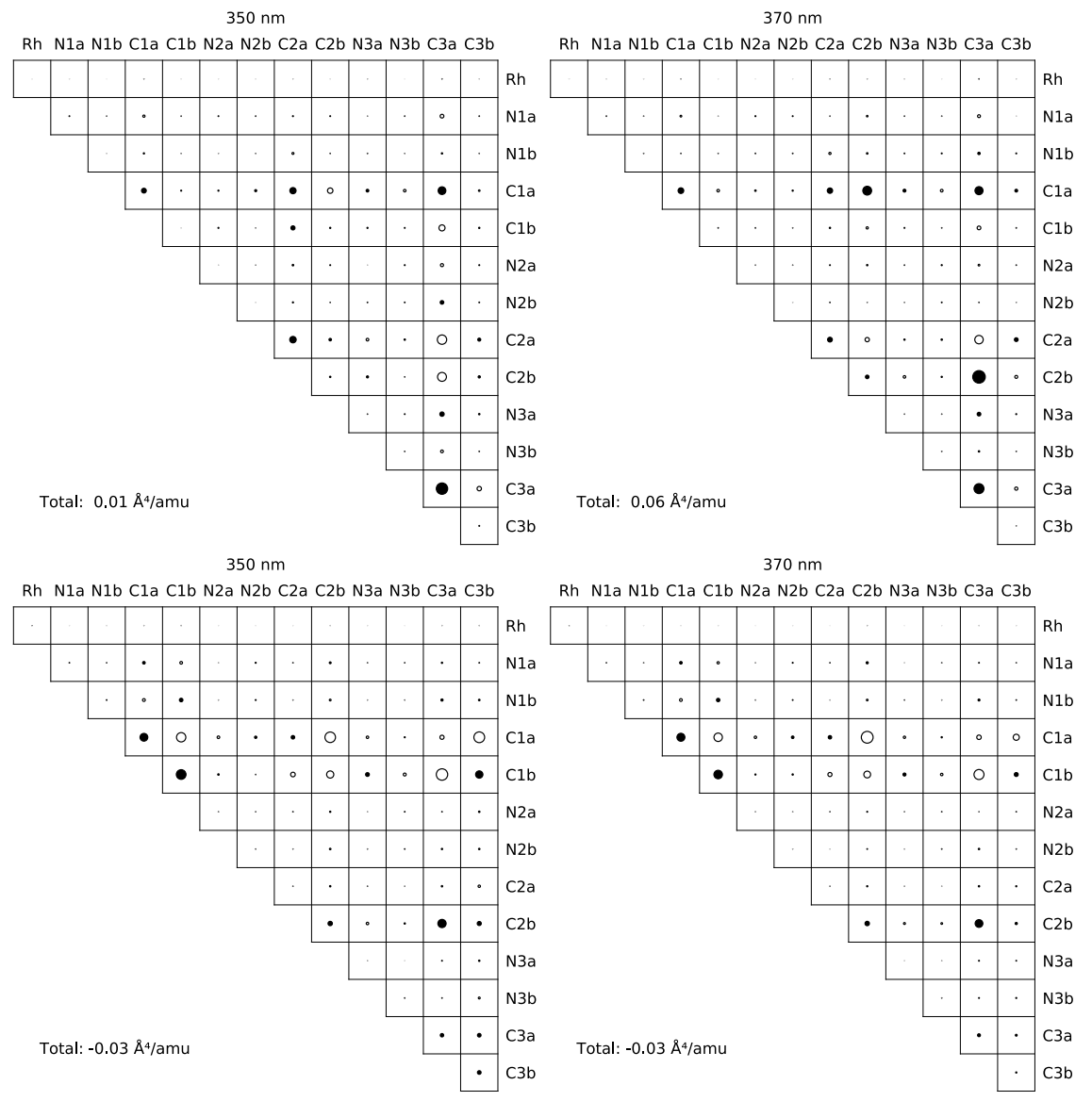

Figure 12: Group coupling matrices for the normal mode at $1494.2 \mathrm{~cm}^{-1}$ (top panel) and the mode at $1496.6 \mathrm{~cm}^{-1}$ (bottom panel) for two different excitation wavelengths (left: $350 \mathrm{~nm}$; right: $370 \mathrm{~nm}$ ). Positive contributions are represented by filled circles, while negative contributions are drawn as empty circles. The radius of the circle is proportional to the size of the corresponding GCM matrix element.

As a final example, it is also instructive to examine the six normal modes representing $\mathrm{NH}_{2}$ scissoring vibrations more closely. As we can see, in these normal modes there are almost only the $\mathrm{NH}_{2}$ groups vibrating. As in the example before, these normal modes are very similar to each other in that they describe basically identical vibrations, and they feature similar wavenumbers. In this case, all six normal modes show the same qualitative trend for different excitation wavelengths: They all feature higher intensities at shorter wavelengths, and lower intensities at longer wavelengths. However, they are affected to different degrees. This is best seen when comparing the two modes at $1658.2 \mathrm{~cm}^{-1}$ and $1664.6 \mathrm{~cm}^{-1}$ with one another. While 
both share about the same maximum intensity, their minimum intensities largely differ. While the first mode is only reduced by a factor of about eight, the intensity of the second mode is affected much more strongly, being reduced by more than a factor of 70 .

In summary, we have seen that a complex electronic structure with several closelying states leads to correspondingly complex (resonance) ROA spectra. While we have several tools at our disposal to analyze these spectra in great detail, it remains difficult to identify simple rules that allow one to predict whether a given normal mode will change a lot in intensity or not under resonance conditions.

\section{Conclusions}

We calculated the (resonance) ROA spectrum of $\left[\mathrm{Rh}(\mathrm{en})_{3}\right]^{3+}$ at a range of different wavelengths. These spectra were then examined, in particular with regard to resonance effects. Since the lowest two electronic excitations are almost equal in energy and also the third lowest excited state is rather close, there are always mul-

tiple states involved in these resonance effects. Therefore, the resulting spectra are never monosignate. Large differences can be observed between the spectrum at fullresonant wavelength and the spectra at off-resonance wavelengths. In many cases, the intensity of a given normal mode is enhanced significantly through these resonances, while other normal modes are almost unaffected or even de-enhanced (this being the consequence of the involvement of multiple electronically excited states). We analyzed two such modes in greater detail by means of the group coupling matrices proposed by Hug. In the case of the first normal mode, which was significantly enhanced under resonance condition, the intensity originated mainly in the $\mathrm{NH}_{2}$ groups and the couplings between them. For larger excitation wavelengths, these contributions became significantly weaker, and concomitantly the intensity of this normal mode decreased. In the case of the second mode, the intensity of which stays almost constant across all excitation wavelengths, the ROA intensity is generated almost equally across all individual groups of the molecule, and no clear pattern can be established.

When analyzing the molecular orbitals of the lowest three electronic excitations, these excitations can all be interpreted as moving electronic charge from the metal center to the $\mathrm{NH}_{2}$ groups. Therefore, many modes which involve $\mathrm{NH}_{2}$ vibrations are strongly enhanced, while many other modes not involving these groups are not. However, there are exceptions to both cases. Therefore, it remains difficult to establish simple but still generally valid rules which would allow one to predict how strongly the intensity of a particular normal mode is affected by resonance effects. 


\section{Supporting Information}

Cartesian coordinates of $\left[\mathrm{Rh}(\mathrm{en})_{3}\right]^{3+}$, investigation of the influence of the damping parameter $\Gamma$, verification of origin independence of the ROA spectra, an example input file, as well as the intensities of all normal modes as a function of the excitation wavelength.

\section{Acknowledgments}

The author would like to thank Prof. M. Reiher (ETH Zurich) for continuous support and many inspiring discussions.

\section{References}

[1] Barron, L. D. Molecular Light Scattering and Optical Activity, 2nd ed.; Cambridge University Press: Cambridge, UK, 2004.

[2] Barron, L. D.; Buckingham, A. D. Rayleigh and Raman Scattering from Optically Active Molecules. Mol. Phys. 1971, 20, 1111-1119.

[3] Barron, L. D.; Bogaard, M. P.; Buckingham, A. D. Raman Scattering of Circularly Polarized Light by Optically Active Molecules. J. Am. Chem. Soc. 1973, 95, 603-605.

[4] Hug, W.; Kint, S.; Bailey, G. F.; Scherer, J. R. Raman Circular Intensity Differential Spectroscopy. The Spectra of $(-)$ - $\alpha$-Pinene and $(+)$ - $\alpha$-Phenylethylamine. J. Am. Chem. Soc. 1975, 97, 5589-5590.

[5] Spencer, K. M.; Freedman, T. B.; Nafie, L. A. Scattered Circular Polarization Raman Optical Activity. Chem. Phys. Lett. 1988, 149, 367-374.

[6] Hecht, L.; Barron, L. D. Recent Developments in Raman Optical Activity Instrumentation. Faraday Discuss. 1994, 99, 35-47.

[7] Hug, W.; Hangartner, G. A Novel High-Throughput Raman Spectrometer for Polarization Difference Measurements. J. Raman Spectrosc. 1999, 30, 841-852.

[8] Barron, L. D.; Hecht, L.; Blanch, E. W.; Bell, A. F. Solution Structure and Dynamics of Biomolecules from Raman Optical Activity. Prog. Biophys. Mol. Biol. 2000, 73, 1-49. 
[9] Barron, L. D.; Hecht, L.; McColl, I. H.; Blanch, E. W. Raman Optical Activity Comes of Age. Mol. Phys. 2004, 102, 731-744.

[10] Barron, L. D.; Zhu, F.; Hecht, L.; Tranter, G. E.; Isaacs, N. W. Raman Optical Activity: An Incisive Probe of Molecular Chirality and Biomolecular Structure. J. Mol. Struct. 2007, 834-836, 7-16.

[11] Barron, L. D.; Buckingham, A. D. Vibrational Optical Activity. Chem. Phys. Lett. 2010, 492, 199-213.

[12] Wu, T.; You, X.-Z.; Bour, P. Applications of Chiroptical Spectroscopy to Coordination Compounds. Coord. Chem. Rev. 2015, 284, 1-18.

[13] Luber, S. Exploring Raman Optical Activity for Transition Metals: From Coordination Compounds to Solids. Biomed. Spectrosc. Imaging 2015, 4, 255-268.

[14] Johannessen, C.; Hecht, L.; Merten, C. Comparative Study of Measured and Computed Raman Optical Activity of a Chiral Transition Metal Complex. ChemPhysChem 2011, 12, 1419-1421.

[15] Luber, S.; Reiher, M. Raman Optical Activity Spectra of Chiral Transition Metal Complexes. Chem. Phys. 2008, 346, 212-223.

[16] Luber, S.; Reiher, M. Intensity-Carrying Modes in Raman and Raman Optical Activity Spectroscopy. ChemPhysChem 2009, 10, 2049-2057.

[17] Luber, S.; Reiher, M. Prediction of Raman Optical Activity Spectra of Chiral 3-Acetylcamphorato-Cobalt Complexes. ChemPhysChem 2010, 11, 1876-1887.

[18] Luber, S.; Reiher, M. Theoretical Raman Optical Activity Study of the $\beta$ Domain of Rat Metallothionein. J. Phys. Chem. B 2010, 114, 1057-1063.

[19] Humbert-Droz, M.; Oulevey, P.; Lawson Daku, L. M.; Luber, S.; Hagemann, H.; Bürgi, T. Where Does the Raman Optical Activity of $\left[\mathrm{Rh}(\mathrm{en})_{3}\right]^{3+}$ Come from? Insight from a Combined Experimental and Theoretical Approach. Phys. Chem. Chem. Phys. 2014, 16, 23260-23273.

[20] Nafie, L. A. Theory of Resonance Raman Optical Activity: the Single Electronic State Limit. Chem. Phys. 1996, 205, 309-322.

[21] Vargek, M.; Freedman, T. B.; Lee, E.; Nafie, L. A. Experimental Observation of Resonance Raman Optical Activity. Chem. Phys. Lett. 1998, 287, 359-364.

[22] Merten, C.; Li, H.; Nafie, L. A. Simultaneous Resonance Raman Optical Activity Involving Two Electronic States. J. Phys. Chem. A 2012, 116, 7329-7336. 
[23] Vidal, L. N.; Giovannini, T.; Cappelli, C. Can the Resonance Raman Optical Activity Spectrum Display Sign Alternation? J. Phys. Chem. Lett. 2016, 7, 3585-3590.

[24] Luber, S.; Neugebauer, J.; Reiher, M. Enhancement and De-Enhancement Effects in Vibrational Resonance Raman Optical Activity. J. Chem. Phys. 2010, 132, 044113.

[25] Jensen, L.; Autschbach, J.; Krykunov, M.; Schatz, G. C. Resonance Vibrational Raman Optical Activity: A Time-Dependent Density Functional Theory Approach. J. Chem. Phys. 2007, 127, 134101.

[26] Baiardi, A.; Bloino, J.; Barone, V. Time-Dependent Formulation of Resonance Raman Optical Activity Spectroscopy. J. Chem. Theory Comput. 2018, 14, 6370-6390.

[27] Merten, C.; Li, H.; Lu, X.; Hartwig, A.; Nafie, L. A. Observation of Resonance Electronic and Non-Resonance-Enhanced Vibrational Natural Raman Optical Activity. J. Raman Spectrosc. 2010, 41, 1563-1565.

[28] Zhu, F.; Isaacs, N. W.; Hecht, L.; Barron, L. D. Raman Optical Activity: A Tool for Protein Structure Analysis. Structure 2005, 13, 1409-1419.

[29] Connelly, N. G., Damhus, T., Hartshorn, R. M., Hutton, A. T., Eds. Nomenclature of Inorganic Chemistry - IUPAC Recommendations 2005; RSC Publishing: Cambridge, UK, 2005.

[30] Valiev, M.; Bylaska, E. J.; Govind, N.; Kowalski, K.; Straatsma, T. P.; Van Dam, H. J. J.; Wang, D.; Nieplocha, J.; Apra, E.; Windus, T. L. et al. NWChem: A Comprehensive and Scalable Open-Source Solution for Large Scale Molecular Simulations. Comput. Phys. Commun. 2010, 181, 1477-1489.

[31] Kohn, W.; Sham, L. J. Self-Consistent Equations Including Exchange and Correlation Effects. Phys. Rev. 1965, 140, A1133-A1138.

[32] Adamo, C.; Barone, V. Toward Reliable Density Functional Methods Without Adjustable Parameters: The PBE0 Model. J. Chem. Phys. 1999, 110, 61586170 .

[33] Weigend, F.; Ahlrichs, R. Balanced Basis Sets of Split Valence, Triple Zeta Valence and Quadruple Zeta Valence Quality for H to Rn: Design and Assessment of Accuracy. Phys. Chem. Chem. Phys. 2005, 7, 3297-3305.

[34] Andrae, D.; Häußermann, U.; Dolg, M.; Stoll, H.; Preuß, H. Energy-Adjusted ab Initio Pseudopotentials for the Second and Third Row Transition Elements. Theor. Chim. Acta 1990, 77, 123-141. 
[35] Weigend, F. Accurate Coulomb-Fitting Basis Sets for H to Rn. Phys. Chem. Chem. Phys. 2006, 8, 1057-1065.

[36] Grimme, S.; Antony, J.; Ehrlich, S.; Krieg, H. A Consistent and Accurate $a b$ Initio Parametrization of Density Functional Dispersion Correction (DFT-D) for the 94 Elements H-Pu. J. Chem. Phys. 2010, 132, 154104.

[37] Weymuth, T.; Haag, M. P.; Kiewisch, K.; Luber, S.; Schenk, S.; Jacob, C. R.; Herrmann, C.; Neugebauer, J.; Reiher, M. MoVIPAC: Vibrational Spectroscopy with a Robust Meta-Program for Massively Parallel Standard and Inverse Calculations. J. Comput. Chem. 2012, 33, 2186-2198.

[38] Mathematica 11.2; Wolfram Research, Inc.: Champaign, IL, 2017.

[39] Hunter, J. D. Matplotlib: A 2D Graphics Environment. Comput. Sci. Eng. 2007, 9, 90-95.

[40] Humphrey, W.; Dalke, A.; Schulten, K. VMD: Visual Molecular Dynamics. J. Mol. Graphics 1996, 14, 33-38.

[41] Stone, J. An Efficient Library for Parallel Ray Tracing and Animation. M.Sc. thesis, Computer Science Department, University of Missouri-Rolla, 1998.

[42] JMOL - An Open-Source Java Viewer for Chemical Structures in 3D, http://www.jmol.org/ .

[43] Becke, A. D. Density-Functional Thermochemistry. III. The Role of Exact Exchange. J. Chem. Phys. 1993, 98, 5648-.

[44] Lee, C.; Yang, W.; Parr, R. G. Development of the Colle-Salvetti CorrelationEnergy Formula Into a Functional of the Electron Density. Phys. Rev. B 1988, 37, 785-789.

[45] Vosko, S. H.; Wilk, L.; Nusair, M. Accurate Spin-Dependent Electron Liquid Correlation Energies for Local Spin Density Calculations: a Critical Analysis. Can. J. Phys. 1980, 58, 1200-1211.

[46] Frisch, M. J.; Trucks, G. W.; Schlegel, H. B.; Scuseria, G. E.; Robb, M. A.; Cheeseman, J. R.; Scalmani, G.; Barone, V.; Mennucci, B.; Petersson, G. A. et al. Gaussian 09 Revision C.1. Gaussian Inc. Wallingford CT 2009.

[47] Herrmann, C.; Ruud, K.; Reiher, M. Can Raman Optical Activity Separate Axial from Local Chirality? A Theoretical Study of Helical Deca-Alanine. ChemPhysChem 2006, 7, 2189-2196.

[48] Hug, W. Visualizing Raman and Raman Optical Activity Generation in Polyatomic Molecules. Chem. Phys. 2001, 264, 53-69. 


\section{TOC Graphic}

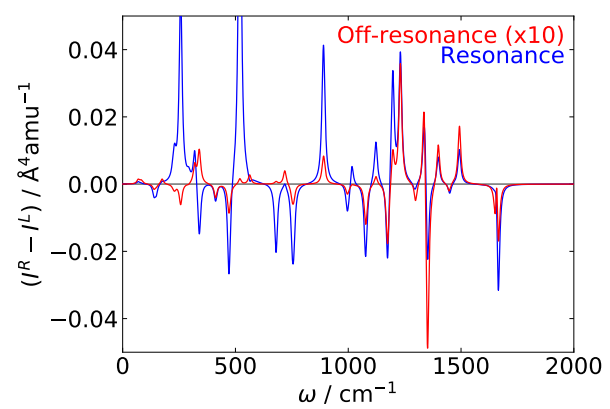




\title{
Resonance Effects in the Raman Optical Activity Spectrum of $\left[\operatorname{Rh}(\mathrm{en})_{3}\right]^{3+}$
}

\author{
Thomas Weymuth ${ }^{a, 1}$ \\ ${ }^{a}$ Laboratory for Physical Chemistry, ETH Zurich, \\ 8093 Zurich, Switzerland
}

September 30, 2019

Supporting Information

\footnotetext{
${ }^{1}$ Corresponding author; e-mail: thomas.weymuth@phys.chem.ethz.ch; ORCID: 0000-0001-71027022
} 


\section{S1 Cartesian Coordinates of $\left[\operatorname{Rh}(\mathrm{en})_{3}\right]^{3+}$}

Below we give the Cartesian coordinates of $\left[\mathrm{Rh}(\mathrm{en})_{3}\right]^{3+}$ as obtained from the structure optimization. All values are given in $\AA$.

\begin{tabular}{|c|c|c|c|}
\hline C & -2.56007083 & -1.35708902 & -0.54082819 \\
\hline $\mathrm{H}$ & -3.10328879 & -2.24869940 & -0.86238831 \\
\hline $\mathrm{H}$ & -3.08660763 & -0.49282315 & -0.95265826 \\
\hline $\mathrm{C}$ & -2.47421399 & -1.28021359 & 0.95943603 \\
\hline $\mathrm{H}$ & -3.46617474 & -1.19818162 & 1.40985356 \\
\hline $\mathrm{H}$ & -2.00345265 & -2.17345255 & 1.37692725 \\
\hline $\mathrm{N}$ & -1.16889899 & -1.35936049 & -1.09006688 \\
\hline $\mathrm{H}$ & -1.21286788 & -1.16089489 & -2.08908158 \\
\hline $\mathrm{H}$ & -0.80398432 & -2.31021408 & -1.04209812 \\
\hline $\mathrm{N}$ & -1.63162263 & -0.09834763 & 1.31900179 \\
\hline $\mathrm{H}$ & -1.37112102 & -0.16978020 & 2.30207886 \\
\hline $\mathrm{H}$ & -2.21364735 & 0.73774283 & 1.27663904 \\
\hline $\mathrm{Rh}$ & -0.00002018 & 0.00010660 & 0.00233207 \\
\hline C & -0.02679481 & 2.88428537 & -0.60574077 \\
\hline $\mathrm{C}$ & 2.35191497 & -1.42093764 & -1.06582865 \\
\hline $\mathrm{H}$ & -0.58752840 & 3.79240567 & -0.83894857 \\
\hline $\mathrm{H}$ & 3.35099977 & -1.38928934 & -1.50663534 \\
\hline $\mathrm{H}$ & 0.88253316 & 2.90273817 & -1.21114681 \\
\hline $\mathrm{H}$ & 1.80857443 & -2.23169584 & -1.55717426 \\
\hline $\mathrm{C}$ & 0.29510901 & 2.80224385 & 0.86182796 \\
\hline $\mathrm{C}$ & 2.41748095 & -1.63037140 & 0.42278229 \\
\hline $\mathrm{H}$ & 0.93417380 & 3.63108091 & 1.17516908 \\
\hline $\mathrm{H}$ & 2.87727091 & -2.59042055 & 0.66869121 \\
\hline $\mathrm{H}$ & -0.61140381 & 2.84628607 & 1.47013288 \\
\hline $\mathrm{H}$ & 3.01413515 & -0.85371141 & 0.90728810 \\
\hline $\mathrm{N}$ & -0.81152322 & 1.66769055 & -0.98163326 \\
\hline $\mathrm{N}$ & 1.61872404 & -0.14367645 & -1.32703344 \\
\hline $\mathrm{H}$ & 1.35244579 & -0.11627064 & -2.31077538 \\
\hline $\mathrm{H}$ & -0.82072619 & 1.58682826 & -1.99789514 \\
\hline $\mathrm{H}$ & 2.27364722 & 0.63115483 & -1.22583125 \\
\hline $\mathrm{H}$ & -1.78870042 & 1.82488763 & -0.73660797 \\
\hline $\mathrm{N}$ & 0.96576365 & 1.49089727 & 1.12047395 \\
\hline $\mathrm{N}$ & 1.02648486 & -1.55627954 & 0.96667280 \\
\hline $\mathrm{H}$ & 0.97308157 & 1.32058012 & 2.12565099 \\
\hline $\mathrm{H}$ & 1.07697248 & -1.45807464 & 1.98016955 \\
\hline
\end{tabular}




$\begin{array}{lrrr}\mathrm{H} & 1.95183719 & 1.57898160 & 0.87656334 \\ \mathrm{H} & 0.57692005 & -2.46074498 & 0.82712764\end{array}$

\section{S2 Damping Factor}

In order to investigate the influence of the damping value, we calculated the ROA spectrum of $\left[\mathrm{Rh}(\mathrm{en})_{3}\right]^{3+}$ for two different damping values. In the first case, we adopted a rather small damping value of 0.0037 a.u.; in the second case, a larger value of 0.008 a.u. was used. For both damping values, we calculated the spectrum with an excitation wavelength of $307.66 \mathrm{~nm}$, i.e., under full-resonance conditions, and with an excitation wavelength of $370 \mathrm{~nm}$. The four resulting spectra are shown in Fig. S1. As one can see, when an excitation wavelength of $370 \mathrm{~nm}$ is adopted, the two different damping values lead to virtually identical spectra, and therefore have no influence ( $c f$. , the top two spectra in Fig. S1). Under full-resonance conditions, however, the damping value has a rather large influence on the overall spectral intensity. With larger damping value, the overall intensity is greatly reduced (up to a factor of about five). However, the relative intensities as well as the overall band shape is much less affected by changes in the damping value. We therefore conclude that the exact numerical value adopted for the damping value is not of primary importance as long as one and the same value is used throughout our study. This will suffice to make the individual spectra comparable to each other. 

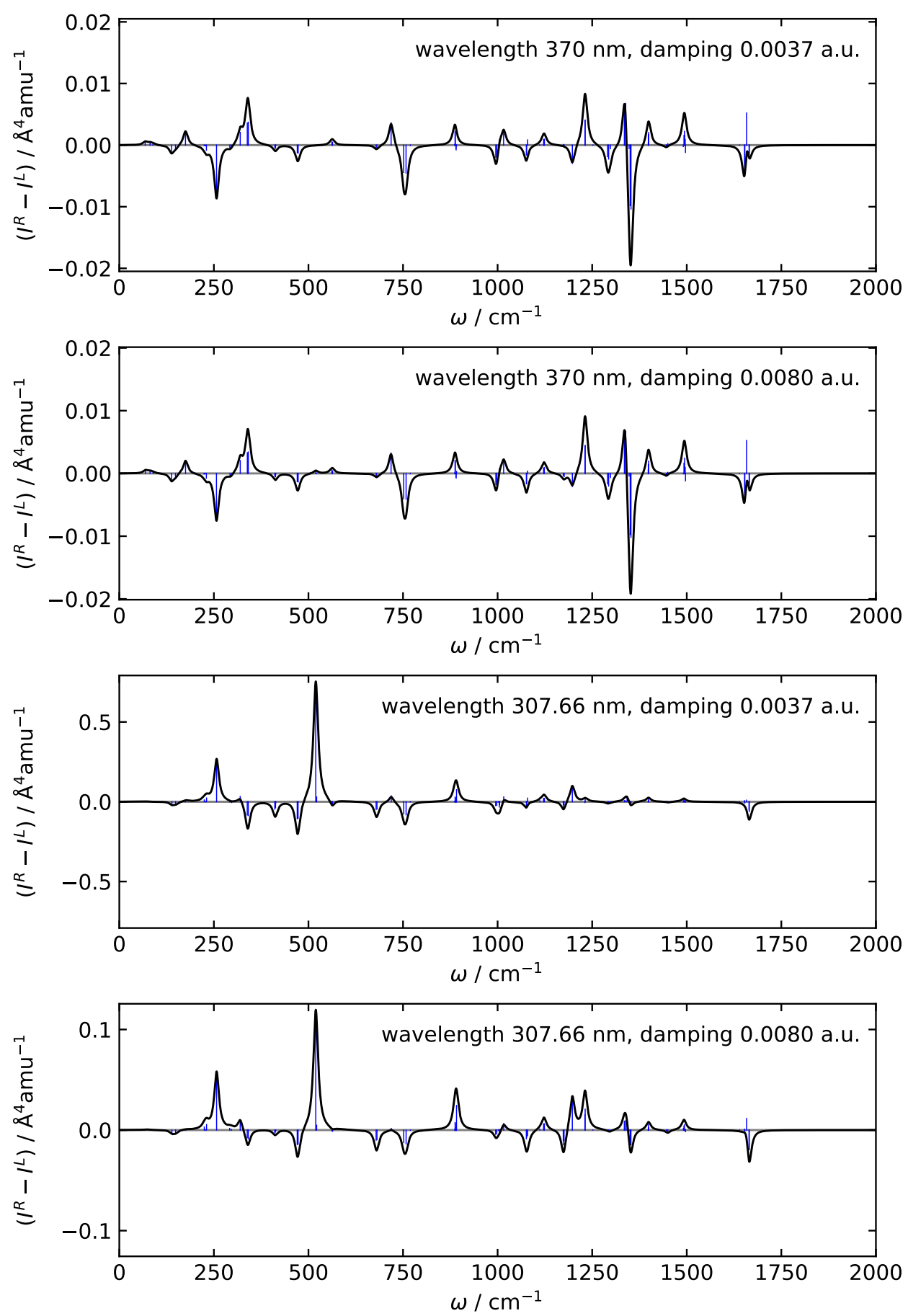

Figure S1: ROA spectra of $\left[\mathrm{Rh}(\mathrm{en})_{3}\right]^{3+}$ for two different excitation wavelengths and two different damping values. A wavelength of $307.66 \mathrm{~nm}$ yields full-resonance spectra. Note that the individual spectra span different intensity ranges. 


\section{S3 Origin Dependence}

In order to check whether the methodology followed in this work yields ROA spectra which are origin independent, we shifted the molecule by $10 \AA$ in each of the three spatial directions and recalculated the spectrum under full-resonance conditions. As can be seen by comparing the first and third spectrum in Fig. S2, the spectrum does literally not change. We therefore conclude that our spectra are not dependent on the choice of origin.

As a further test, we also calculated the ROA spectrum under full-resonance conditions without gauge-including atomic orbitals (GIAOs), both with the molecule at its original location and with the molecule shifted by $10 \AA$ in all three spatial directions. As can be seen from Fig. S2, in our case the use of GIAOs apparently has no observable effects on the ROA spectrum. 

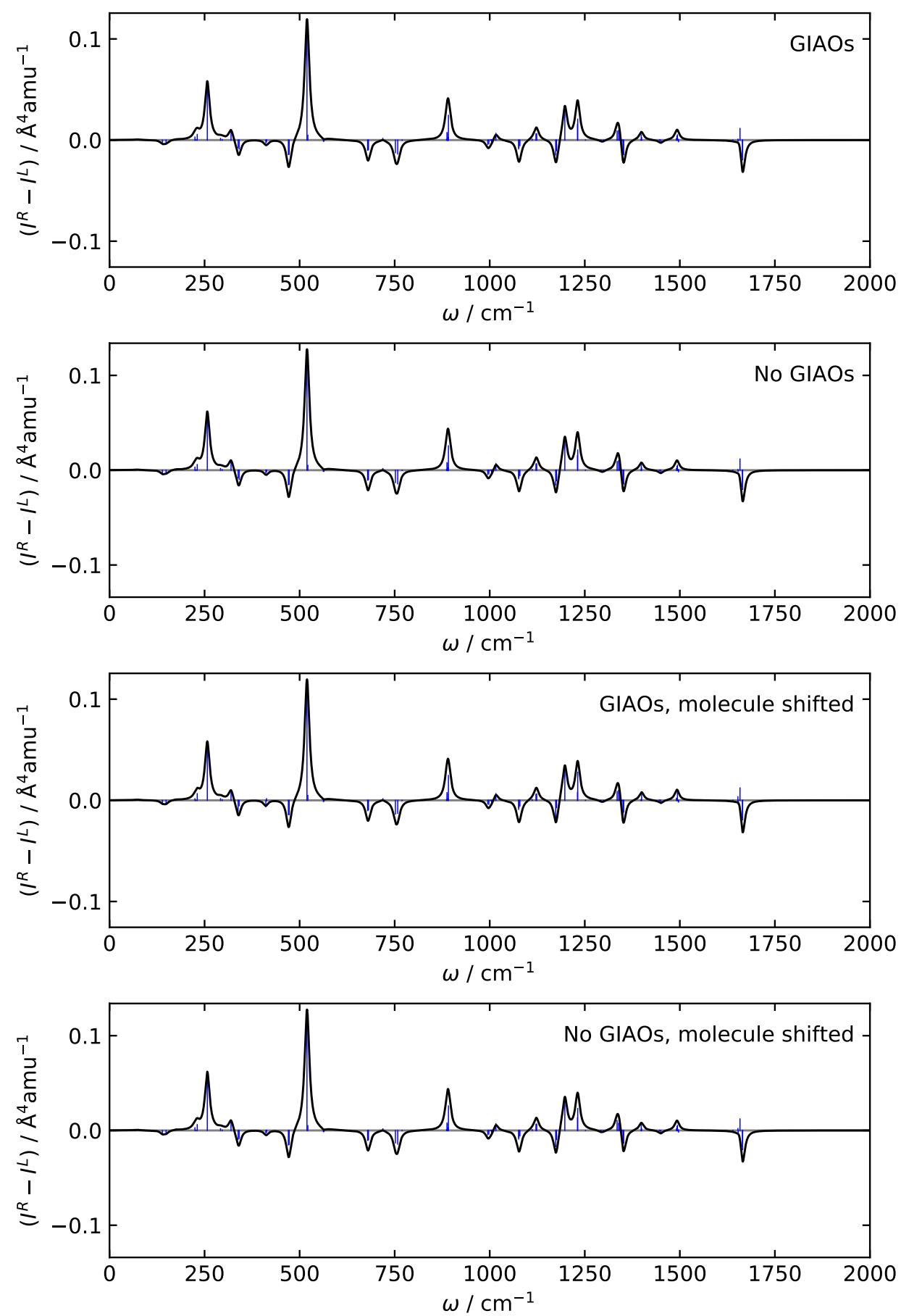

Figure S2: ROA spectra of $\left[\mathrm{Rh}(\mathrm{en})_{3}\right]^{3+}$ calculated either with or without gauge-including atomic orbitals. In the upper two spectra, the molecule is placed very close to the origin, while in the lower two spectra, the molecule is shifted by $10 \AA$ in all three spatial directions. 


\section{S4 Example Input File}

Below we give an example NWCHEM input file as used in this work to calculate the ROA spectra. The nuclear coordinates have been omitted in this example; in the actual calculations, the distorted structures as generated by MoVIPAC have been inserted.

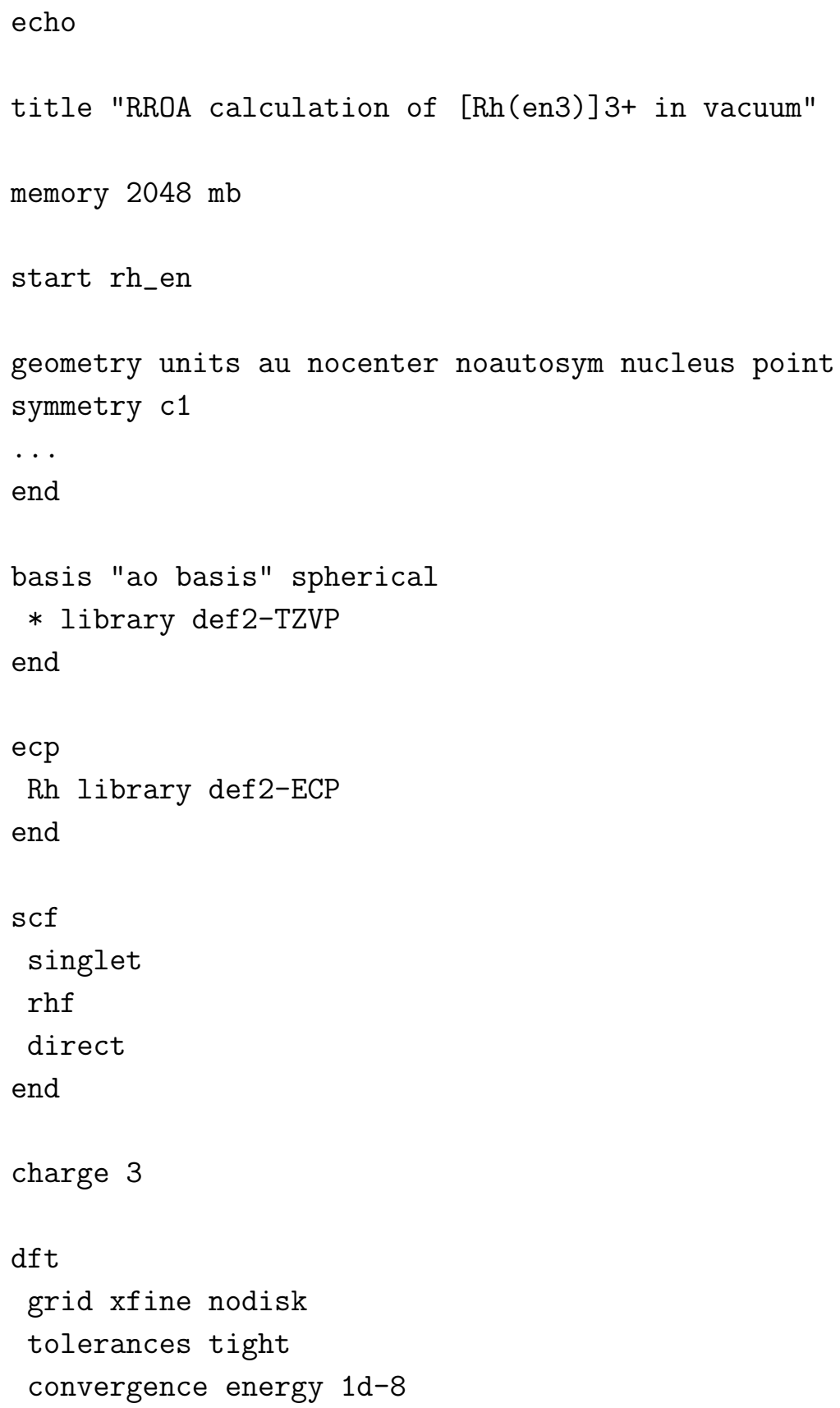




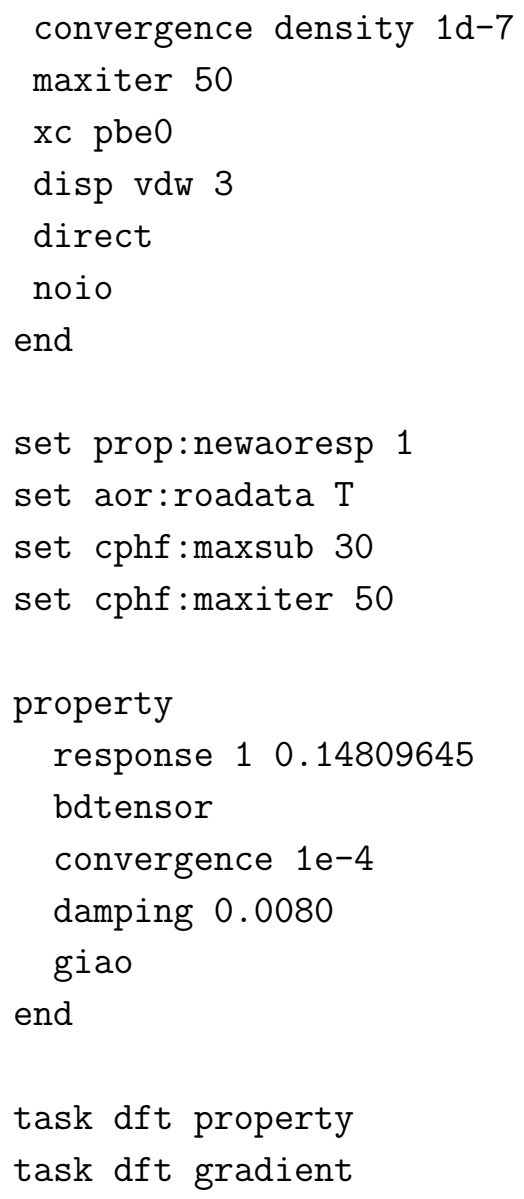




\section{S5 Intensities of Normal Modes}
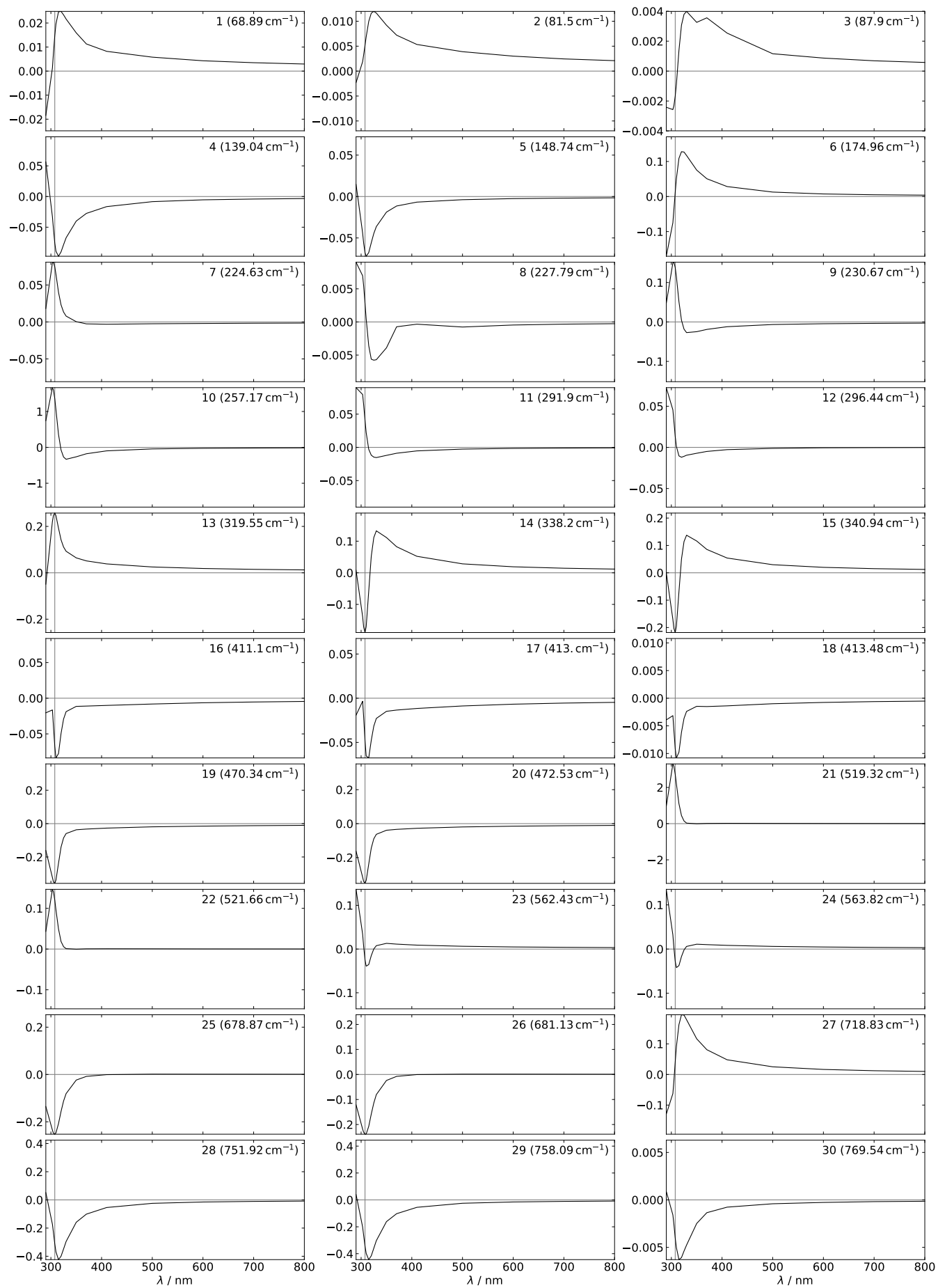

Figure S3: Intensities of normal modes 1 to 30 as a function of excitation wavelength. All intensities are given in $\AA^{4} / \mathrm{amu}^{-1}$. The vertical line highlights the full-resonance wavelength. 


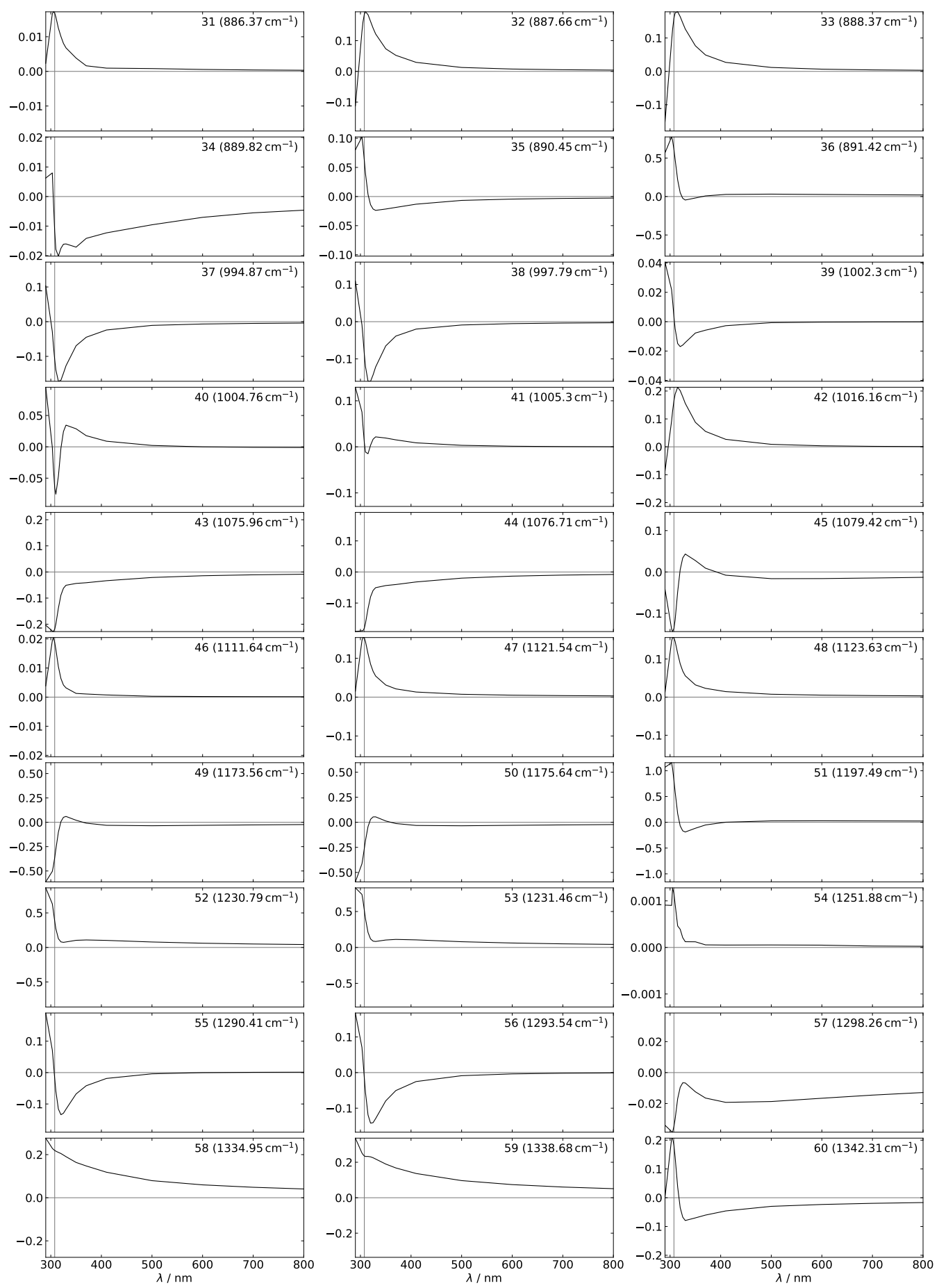

Figure S4: Intensities of normal modes 31 to 60 as a function of excitation wavelength. All intensities are given in $\AA^{4} / \mathrm{amu}^{-1}$. The vertical line highlights the full-resonance wavelength. 

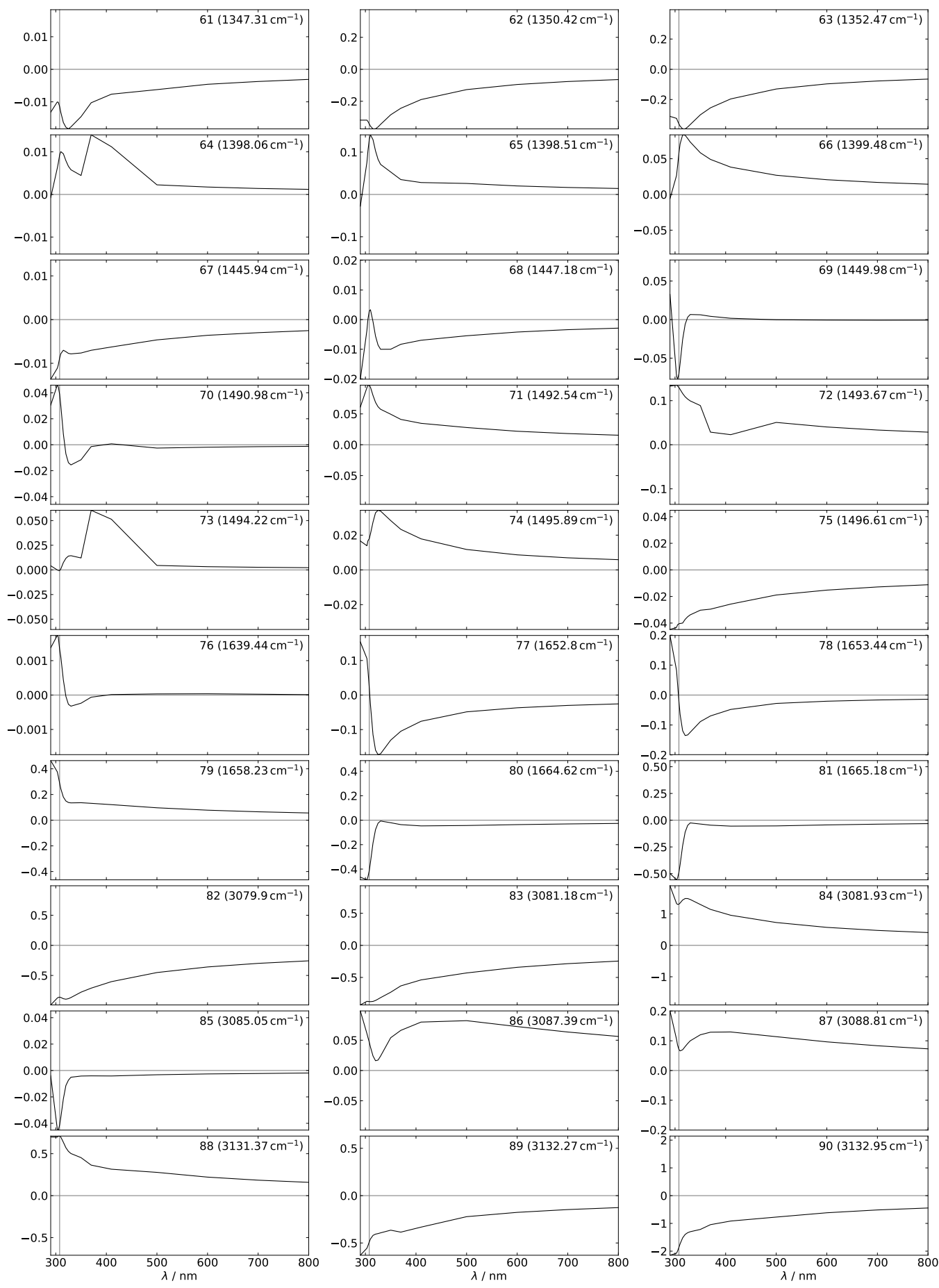

Figure S5: Intensities of normal modes 61 to 90 as a function of excitation wavelength. All intensities are given in $\AA^{4} / \mathrm{amu}^{-1}$. The vertical line highlights the full-resonance wavelength. 

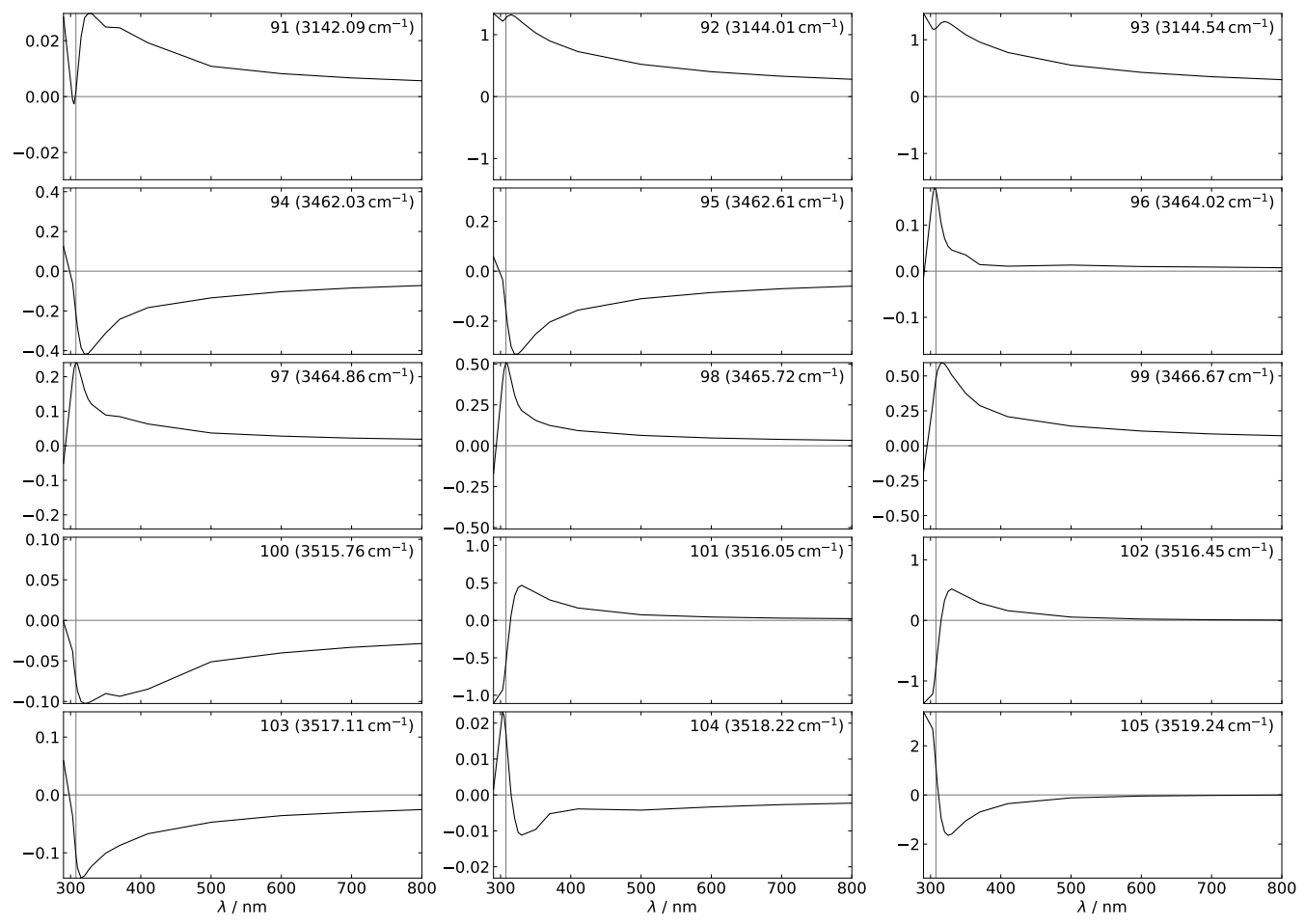

Figure S6: Intensities of normal modes 91 to 105 as a function of excitation wavelength. All intensities are given in $\AA^{4} / \mathrm{amu}^{-1}$. The vertical line highlights the full-resonance wavelength. 